\title{
Identification and Modeling of the Airbrake of an Experimental Unmanned Aircraft
}

\author{
Peter Bauer $^{1}$ (D) - Lysandros Anastasopoulos ${ }^{2} \cdot$ Franz-Michael Sendner $^{3} \cdot$ Mirko Hornung $^{2} \cdot$ Balint Vanek $^{1}$
}

Received: 19 October 2019 / Accepted: 15 April 2020 / Published online: 6 June 2020

(C) The Author(s) 2020

\begin{abstract}
This paper presents the modeling, system identification, simulation and flight testing of the airbrake of an unmanned experimental aircraft in frame of the FLEXOP H2020 EU project. As the aircraft is equipped with a jet engine with slow response an airbrake is required to increase deceleration after speeding up the aircraft for flutter testing in order to remain inside the limited airspace granted by authorities for flight testing. The airbrake consists of a servo motor, an opening mechanism and the airbrake control surface itself. After briefly introducing the demonstrator aircraft, the airbrake design and the experimental test benches the article gives in depth description of the modeling and system identification referencing also previous work. System identification consists of the determination of the highly nonlinear (saturated and load dependent) servo actuator dynamics and the nonlinear aerodynamic and mechanical characteristics including stiffness and inertia effects. New contributions relative to the previous work are a unified servo angular velocity limit model considering opening against the load or closing with it, the detailed construction and evaluation of airbrake normal and drag force models considering the whole deflection and aircraft airspeed range, the presentation of a unified aerodynamic - mechanic nonlinearity model giving direct relation between airbrake angle, dynamic pressure and servo torque and the transfer function-based modeling of stiffness and inertial effects in the mechanism. The identified servo dynamical model includes system delay, inner saturation, the aforementioned load dependent angular velocity limit model and a transfer function model. The servo model was verified based-on test bench measurements considering the whole opening angle and dynamic load range of the airbrake. New, unpublished measurements with gradually increasing servo load as the servo moves are also considered to verify the model in more realistic circumstances. Then the full airbrake model is constructed and tested in simulation to check realistic behavior. In the next step the airbrake model integrated into the nonlinear simulation model of the FLEXOP aircraft is tested by flying simulated test trajectories with the baseline controller of the aircraft in software-in-the-loop (SIL) Matlab simulation. First, the standalone airbrake simulation is compared to the SIL results to verify flawless integration of airbrake model into the nonlinear aircraft simulation. Then deceleration times with and without airbrake are compared underlining the usefulness of the airbrake in the test mission. Finally, real flight data is used to verify and update the airbrake model and show the effectiveness of the airbrake.
\end{abstract}

Keywords Aircraft airbrake $\cdot$ Dynamic test bench $\cdot$ System identification $\cdot$ Simulation $\cdot$ Flight test results

The research leading to these results is part of the FLEXOP project. This project has received funding from the European Unions Horizon 2020 research and innovation program under grant agreement No 636307. Part of this research has received funding from the European Union's Horizon 2020 research and innovation programme under grant agreement No 815058 (FLiPASED project).

Peter Bauer

bauer.peter@sztaki.hu

1 Systems and Control Laboratory, Institute for Computer Science and Control (SZTAKI), Kende utca 13-17, Budapest, H-1111, Hungary

\section{Introduction}

The FLEXOP EU H2020 research project [2] targeted to develop an experimental aircraft (see Fig. 1) with interchangeable wings (a rigid, a flexible and an aeroelastically

2 Department of Mechanical Engineering, Institute of Aircraft Design, Technical University of Munich (TUM), Boltzmannstr. 15, Garching b., Munich, 85748, Germany

3 Research \& Technology, FACC Operations GmbH, Breitenaich 52, Sankt Martin im Innkreis, A-4973, Austria 
Fig. 1 FLEXOP experimental aircraft (courtesy Technical University Munich)

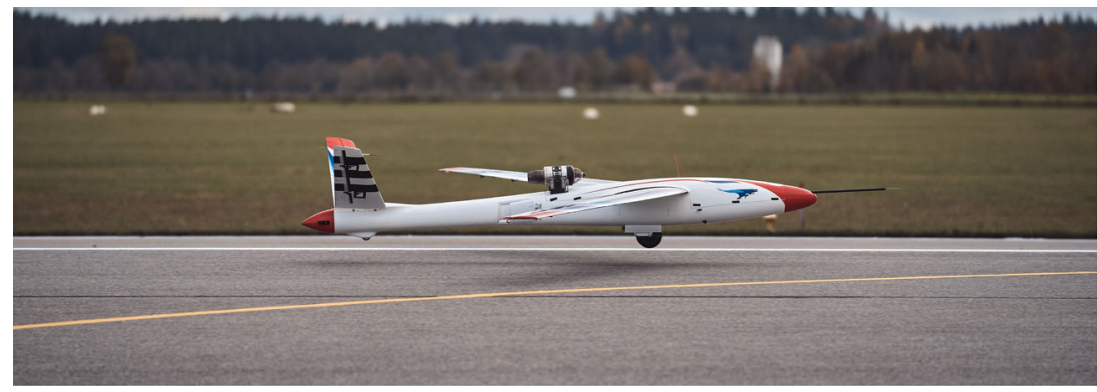

tailored) to test modeling and control possibilities of wing flutter and extend the flutter limit speed with active control.

During the planned test flights the aircraft should fly a racetrack pattern (see Fig. 3) with two turns and two straight sections. One of the straight sections is the test leg where the aircraft should speed up to a given higher reference speed and then also slow down. As it has a BF Turbines BF300 jet engine with slow dynamics ( $5 \mathrm{~s}$ run-up time from idle to full power) a high bandwidth additional actuator is required to make higher decelerations possible. To satisfy this requirement an airbrake was designed [16]. As this will be a critical component of control during flutter tests the precise knowledge of its dynamics is crucial. That's why two test benches were developed by Technical University of Munich (TUM). One to test the whole airbrake mechanism under load including the servo motor, the opening linkages and the airbrake with artificial aerodynamical load [9]. The second is to test the dynamics of the servo motor with different load levels [4]. A preliminary identification of the system dynamics was done in [9] which is extended and refined with several new contributions in this article. Complete identification of the airbrake requires to mathematically model all of the static (such as the ratio from airbrake torque to servo torque) and dynamic characteristics (such as servo motor dynamics).

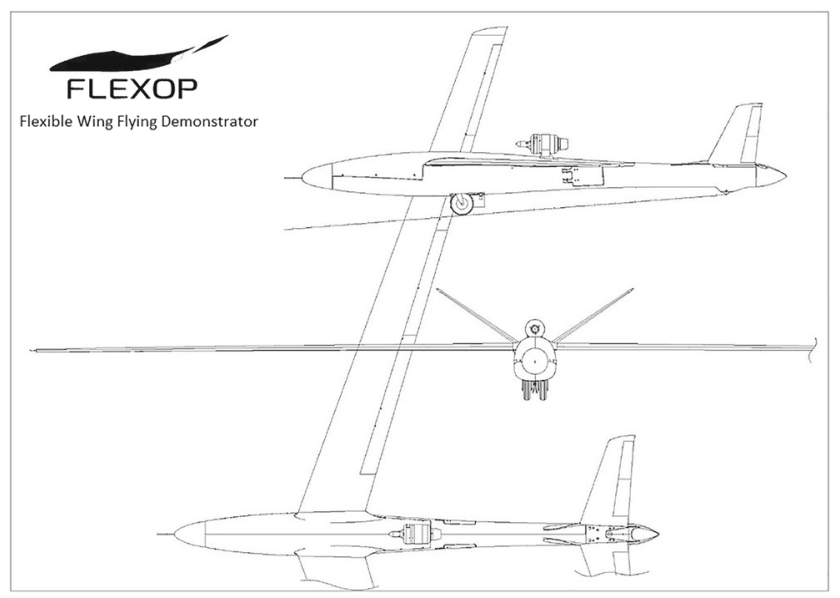

Fig. 2 Three view drawing of the demonstrator aircraft
Considering the literature there are limited sources about servo dynamics identification and neither of them considers full airbrake dynamics together with aerodynamic loads. [3] deals with the identification and control of a direct current (DC) motor arriving to a first order plus integrator plus delay transfer function model. Multi parametric optimization is used to obtain the unknown parameters and uncertainty ranges are also considered. [5] reduces the problem of alternating current (AC) motor system identification to the identification of two unknown parameters as others are known. They are determined through a genetic algorithm. [13] identifies first the linear characteristics of the related mechanism then it identifies a 4th order linear model and a nonlinear model considering servo speed limitation. It finally reduces the required five unknown parameters to three and identifies them with least squares optimization. The nonlinear model proves to better approximate the system. [8] deals with identification and control together. It identifies the DC servo dynamics with third order dynamics plus delay and then a controller is designed. Finally, [18] identifies the dynamics of a DC servo motor considering its second order dynamics and the possible inner controller structures also as usually off-the-self servo motors include control electronics too. Finally, it derives a two transfer functions (TFs) model relating reference and rotation angles to servo torque.

In our case several factors should be considered to have a realistic model of the airbrake and several improvements are offered relative to previous work of the FLEXOP team. This is summarized below.

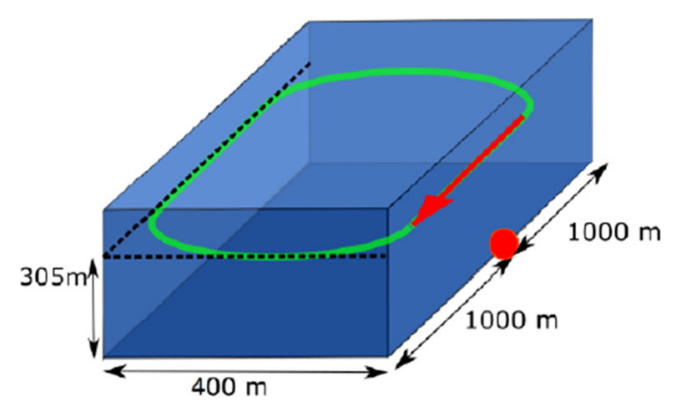

Fig. 3 Flight test pattern 
1. Identification of the servo dynamics itself is a challenge as the literature sources also show. First a servo test bench was developed and measurements with doublet series servo angle references on different frequencies and with different load torque levels were carried out (see [4] and [9]). In our case system identification is complicated by saturation in the inner servo controller (control saturation also considered in [13]) and load dependent maximum angular velocities of the servo which differ if servo travels against or with the load (similar load issue is considered in [18]). This issue is partly covered in [9] but a simpler and unified model is derived in the current work. Pulling out all the nonlinearities and system delay (also identified) finally, a transfer function model of the servo is identified using the output error method [11]. As the measurements show a load dependent steady-state servo angle change in the system output (missing I term in servo control) a separate transfer function model was identified for this and the need for this term verified based-on real flight behavior. This is also a new contribution.

2. The nonlinear aerodynamics is covered by [9] only for $60 \mathrm{~m} / \mathrm{s}$ maximum flight speed and only for the normal force. Regarding that model the addition of the drag coefficient and the drawing of the opening angleairspeed-servo load characteristic is the contribution of this paper.

3. Regarding the nonlinearity in the opening mechanism described in [9] (examined also in [13] for another system) the contribution of the current paper is the construction of a unified aerodynamic plus mechanic nonlinear model to determine a direct relation between airbrake angle, dynamic pressure and servo torque. The precision of this model is also analysed.

4. A final challenge is the stiffness of the mechanism well analysed in [9]. Our contribution here is the transfer function-based frequency domain analysis of system dynamics considering stiffness and airbrake inertia.

The complete servo model was verified based on the test bench measurements (adding new variable load measurements to the previous work) and a full airbrake model was created after including servo motor model, opening mechanism and aerodynamic nonlinearity, stiffness and load dependent steady-state servo angle change. This model was first tested for realistic behaviour in Matlab simulations then integrated into the nonlinear simulation model of the FLEXOP aircraft and tested with software-inthe-loop 'flights' of the test pattern with baseline trajectory tracking controller (see [14]) applying airbrake opening during aircraft deceleration after the test leg. Finally, real flight measured position data of the airbrake is compared to the simulation model (driven by in-flight logged dynamic pressure and deflection command) output to verify the identified model. The airbrake effect on aircraft deceleration was also examined based-on the change in specific energy of the system.

The structure of the current paper is as follows. Section 2 briefly introduces the test aircraft and the design of the airbrake then Section 2.1 summarizes the overall concept of airbrake test and identifiaction. After that Section 3 gives brief information about the test benches. Section 4 presents the identification of the nonlinear airbrake dynamics, Section 5 introduces the full airbrake model with SIL simulation results in Section 5.1 and real flight test results in Section 5.2. Finally, Section 6 concludes the paper.

\section{Airbrake Conceptual Design}

Before discussing airbrake conceptual design the FLEXOP demonstrator aircraft and its operational circumstances should be briefly introduced to clarify the need and the requirements related to the airbrake. The general configuration of the FLEXOP demonstrator is of a conventional design asserting close similarity to state-ofthe-art commercial aircraft. An illustrative three view of the actual demonstrator is depicted in Fig. 2 together with main technical data in Table 1 (regarding the flutter speed see [17]). The high aspect ratio wings feature a moderate leading edge sweep to account for the characteristic bendtwist-coupling of high-subsonic planform designs. The final goal with the demonstrator is to prove possibility of flying beyond flutter onset speed applying active control of the wing dynamics. To isolate wing aeroelastics from thrust interferences and aerodynamic disturbances, an engine installation separated from the wings (on top of the fuselage) was chosen. Also a V-Tail for low-interference drag, as well as for minimum wetted area fuselage was applied. The required disturbance tolerance covers tolerance of moderate turbulence and deterministic wind disturbances until $5-10 \mathrm{~m} / \mathrm{s}$ as the $65 \mathrm{~kg}$ mass of the aircraft make it a small one relative to the usual GA or larger aircraft. In future flutter control tests as turbulence and wind free weather as

Table 1 FLEXOP demonstrator technical data

\begin{tabular}{ll}
\hline Wingspan & $7 \mathrm{~m}$ \\
Wing area & $2.45 \mathrm{~m}^{2}$ \\
Aspect ratio & 20 \\
Leading edge sweep & $20^{\circ}$ \\
Takeoff mass & $65 \mathrm{~kg}$ \\
Cruise speed & $38 \mathrm{~m} / \mathrm{s}$ \\
Flutter onset speed & $51 \mathrm{~m} / \mathrm{s}$ \\
Max engine thrust & $300 \mathrm{~N}$ \\
\hline
\end{tabular}


possible is required to remove additional disturbances of the structural dynamics.

As the test airfield for the demonstrator is München Oberpfaffenhofen all operations should obey German airspace regulations. According to these regulations flight testing within the visual line of sight of a human pilot is required. The flight altitude is limited to a maximum of $305 \mathrm{~m}$ above ground level due to the procedures of the airfield operator. A maximum distance between pilot and vehicle in the order of $1000 \mathrm{~m}$ was considered to provide the required visibility (considering the $7 \mathrm{~m}$ wingspan of the demonstrator) and so a flight test pattern as illustrated in Fig. 3 is assumed. In the figure the position of the pilot is marked with a red dot, with the resulting straight leg for aeroelastic (flutter) test phases marked by a red arrow [15].

Assuming $38-40 \mathrm{~m} / \mathrm{s}$ cruise speed and considering the $1000 \mathrm{~m}$ maximum distance first estimations indicate an available straight distance of $1500 \mathrm{~m}$ for the acceleration to test speed, test of flutter controller and deceleration to a safe airspeed for a turn maneuver. According to preliminary studies on flutter onset, an airspeed range up to $60 \mathrm{~m} / \mathrm{s}$ is required. To account for the resulting requirements on the acceleration and deceleration performance of the vehicle, propulsion alternatives and aerodynamic configurations were studied in a point mass simulation, as illustrated in Fig. 4.

Regarding the engine selection reciprocating, turbojet, turboprop and electric propulsion concepts were studied. Optimization towards minimum system weight (including fuel or battery), complexity and costs finally led to the selection of a micro turbojet engine. The BF Turbines
BF300 engine features a nominal thrust of $300 \mathrm{~N}$ at ISA sea level conditions. The run-up from idle to full throttle consumes 5s. Despite of the unfavorable time constant and the increased consumption, the excellent thrust-to-weight ratio of the engine itself compensates for the additional fuel mass and slow throttle-up however, this arose the need for an additional deceleration device.

The airbrake design was optimized based-on the simple point mass simulation of the demonstrator (see Fig. 4) using a thrust dynamics model based on actual engine measurement data. Historical, empirical data was used to determine preliminary size and estimate drag force for the airbrake. To avoid aerodynamic disturbances or structural buffeting response, wing mounted devices are not applicable to the present problem - although spoilers can provide twice the effectiveness of fuselage installations (see [6]). Thus feasible fuselage locations were evaluated and maximum available airbrake areas determined. An integral solution, merging ventral brake flap and the landing gear door, as well as a double flap system, arranged symmetrically to the fuselage aft of the wing were studied in detail targeting to have a drag force vector going through the center of gravity of the aircraft (see Fig. 5).

With preliminary, conservative assumptions for the brake drag and the actuation speed, a minimum required brake flap area was determined with the help of the point mass simulation of the vehicle. This area estimation indicated integration problems for the ventral solution, since no ground clearance margin was left for the landing gear configuration. So in the next step trade studies for flap aspect ratio, reference area and actuator type were evaluated

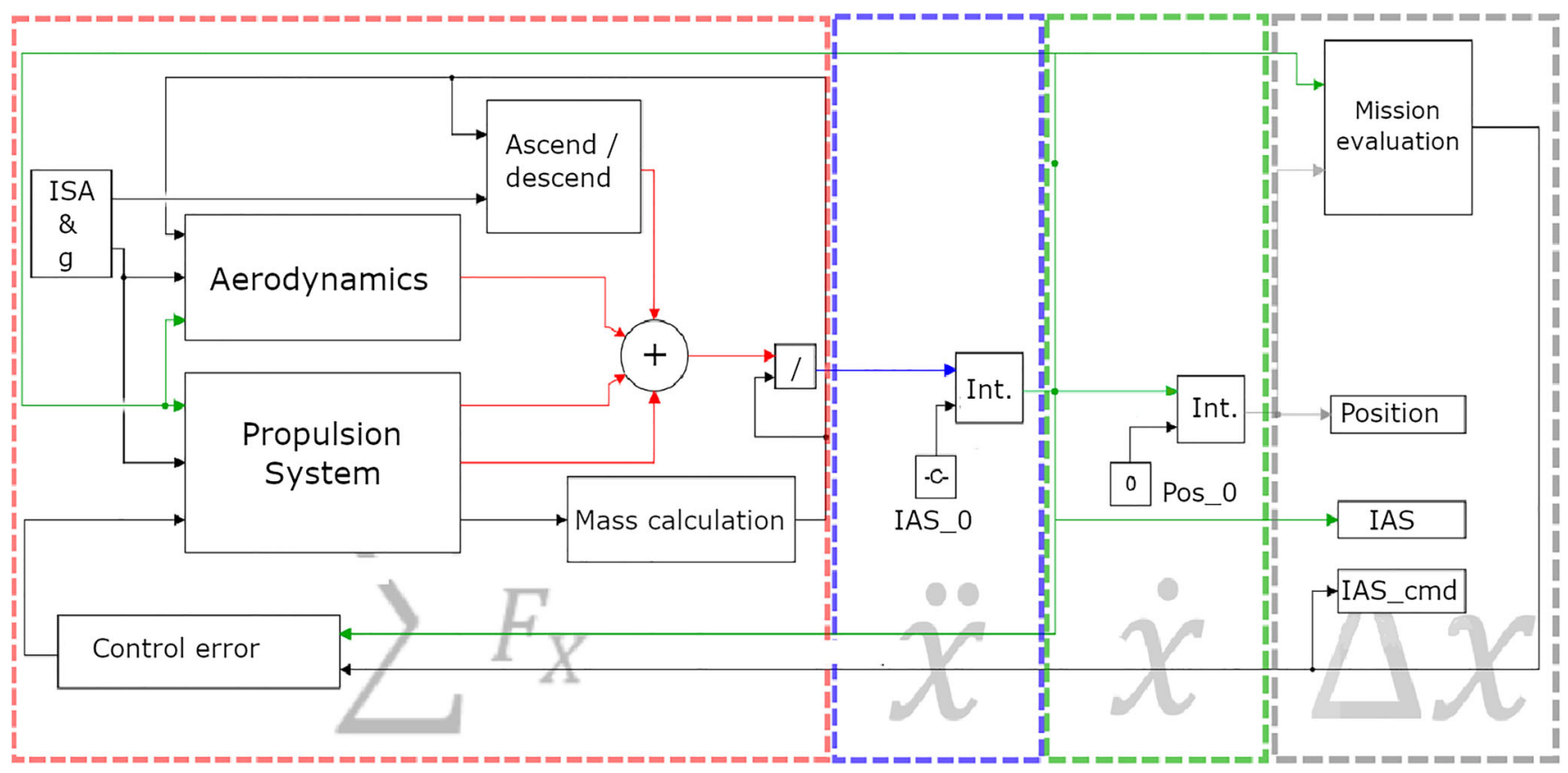

Fig. 4 Block scheme of simple point mass simulation 
Fig. 5 Ventral and symmetric side-by-side arrangement of the brake flaps (from left to right)
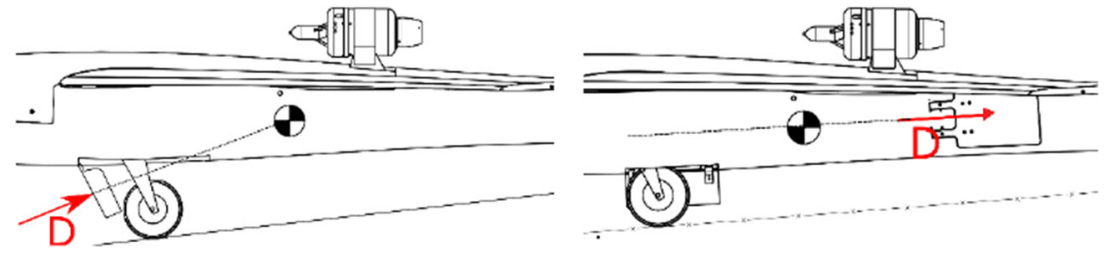

for the side-by-side flap configuration. A simple actuator model assuming either a linear or a rotary actuator was derived based on manufacturer specifications. Dead time and actuator dynamics were neglected. A simple transmission ratio rule was used for the preliminary models. The transmission concept is shown in Fig. 6.

Both linear and rotary models were integrated into the point mass vehicle simulation to evaluate the best fitting concept. At maximum airspeed, the total deflection time accounted to $3.3 \mathrm{~s}$ for the linear actuator and below $0.5 \mathrm{~s}$ for the rotary actuator. In consequence, only a rotary actuator could provide the required short deceleration distance as rendered previously (for details see [16]). So, finally the side-by-side flap configuration and a KST X30-12-150 rotary servo actuator were selected as the main components of the airbrake system. After implementation of the whole system in a mock-up (see Fig. 8) and satisfactory test results installation into the demonstrator fuselage was done. However, to study effects on system dynamics and to tune on-board controllers a mathematical simulation model of the airbrake was required the construction and identification of which is the main topic of this article as follows.

\subsection{Airbrake System Identification Concept}

The identification of the airbrake is a complex task as several parameters and characteristics should be determined. The final construction of the airbrake consists of the servo motor, the opening mechanism and the airbrake itself (see Fig. 37). Going from the inner part to the outer first, the dynamics of the servo motor was determined by building a servo motor test bench (for details see Section 3.2) with a load motor which can be configured to apply different torque loads (consant / varying) on the shaft of the servo. Different dynamic deflections of the servo with different loads were done to cover the whole operational range (see Section 4.1 for the identification of servo dynamics).
After identification the servo model was verified based-on test bench measurements. Second the aerodynamic characteristics of the airbrake were determined including the generated drag force which slows down the aircraft and the load torque on the airbrake shaft resulting from the aerodynamic load. As wind tunnel testing is time consuming and expensive and there is exhaustive literature about such characteristics $[6,12]$ the aerodynamics was finally determined based-on published formulas (see Section 4.2). Finally, the static characteristics were determined such as the ratio of the mechanism from airbrake torque to servo torque and the stiffness of the whole mechanism as this can have a significant effect on the dynamics. The ratio was determined based-on geometrical measurements, however the stiffness required to build a complete airbrake mock-up including the servo motor, the opening mechanism and the airbrake itself with the possibility to add different weights to simulate the aerodynamic loads (for details see Section 3.1). Measuring the deformations with different loads gave the opportunity to determine the opening angle dependent stiffness of the mechanism (see Section 4.2 for the static characteristics). An additional task was to determine the dynamic effect of opening mechanism stiffness and airbrake flap inertia on the overall dynamics. After constructing and identifying all of these model components and building the whole airbrake model the next task was to verify it in Software-in-the-loop (SIL) simulation and on real flight data. This is summarized in Section 5.

\section{Actuator Test Benches}

To make determination of airbrake static and dynamic characteristics possible two test benches were built one is a full size mock-up which also serves to test the whole airbrake opening mechanism together with the servo motor before installing it on the aircraft. The second is a separate
Fig. 6 Conceptual sketches of linear and rotary actuation (from left to right)
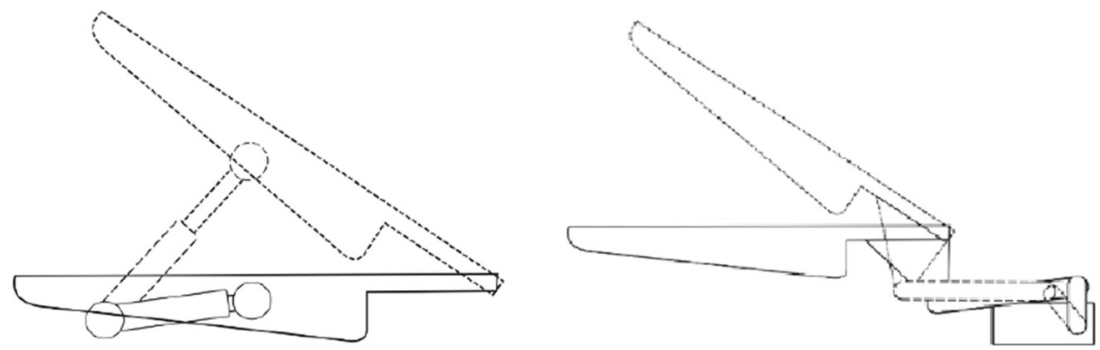
Fig. 7 Side and isometric view of the airbrake mock-up design (from left to right)

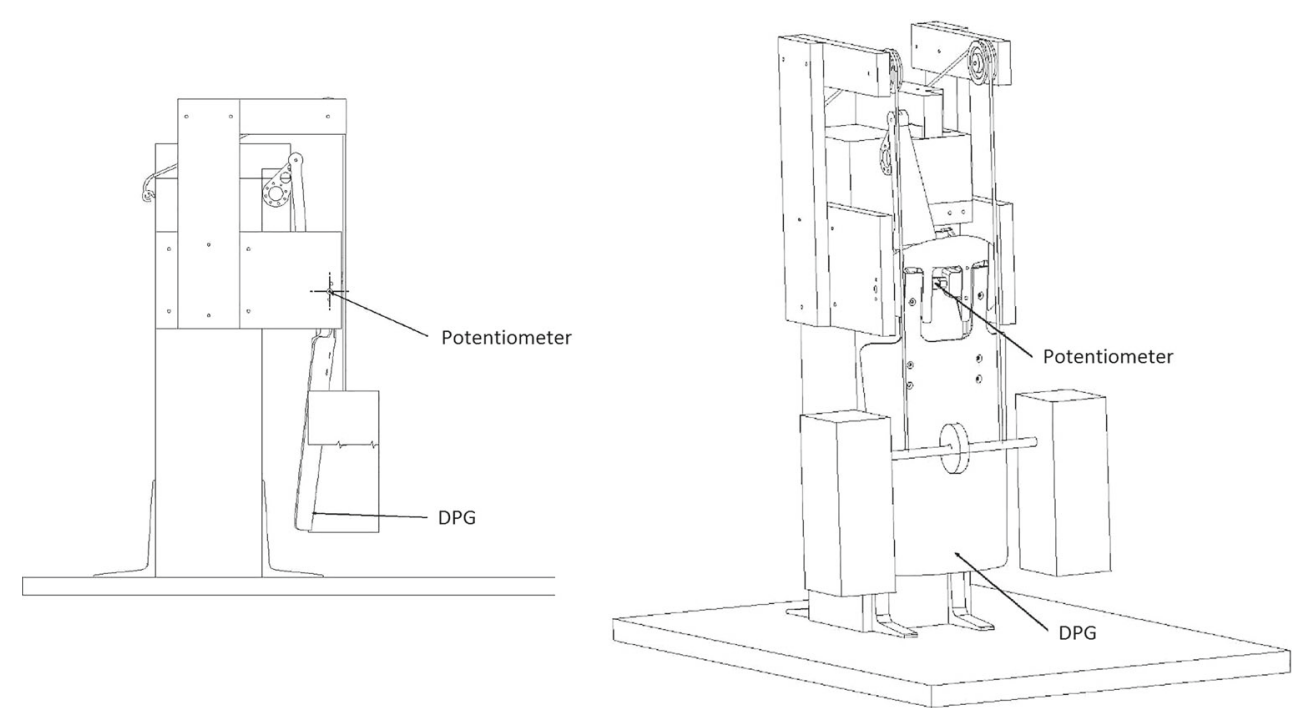

test bench for the servo motor applying an electric load motor to simulate airbrake loading on the servo axle.

\subsection{Full Size Airbrake Mock-Up}

A mass-loaded mock-up of the airbrake installation is used to statically determine the full system stiffness over the range of deflection angles, as well as the static electric power consumption. The mock-up consists of the actual levers and rods, the airbrake flap, the servo actuator as well as a mounting rig. The servo actuator is powered by a laboratory power supply, limited to $20 \mathrm{~A}$. The voltage is set to a constant value of $12 \mathrm{~V}$. The stiffness of the rig is designed such that the angular error induced by the deflection of the assembly under maximum load accumulates to $0.0023^{\circ}$ giving minimal distortion in the deflection measurement of the airbrake itself. A Digital Pitch Gauge (DPG), as well as a potentiometer is used for the determination of the deflection. As the maximum airspeed induced by the angular velocity of the airbrake flap is negligible relative to the $38-60 \mathrm{~m} / \mathrm{s}$ speed of the aircraft no unsteady correction of the aerodynamic forces was required to be applied. So a simple mass carriage/swing was used to solely apply a force normal to the airbrake. Within the quasi-linear load range up to $40^{\circ}$, a mass of $12.658 \mathrm{~kg}$ is used to simulate the aerodynamic load. For the range up to $60^{\circ}$, the mass ballast was adapted to match with the higher aerodynamic load (for details see [9]). The drawings of the mock-up are shown in Fig. 7 while the photos can be seen in Fig. 8.
Fig. 8 Airbrake mock-up with closed and fully opened airbrake (from left to right)
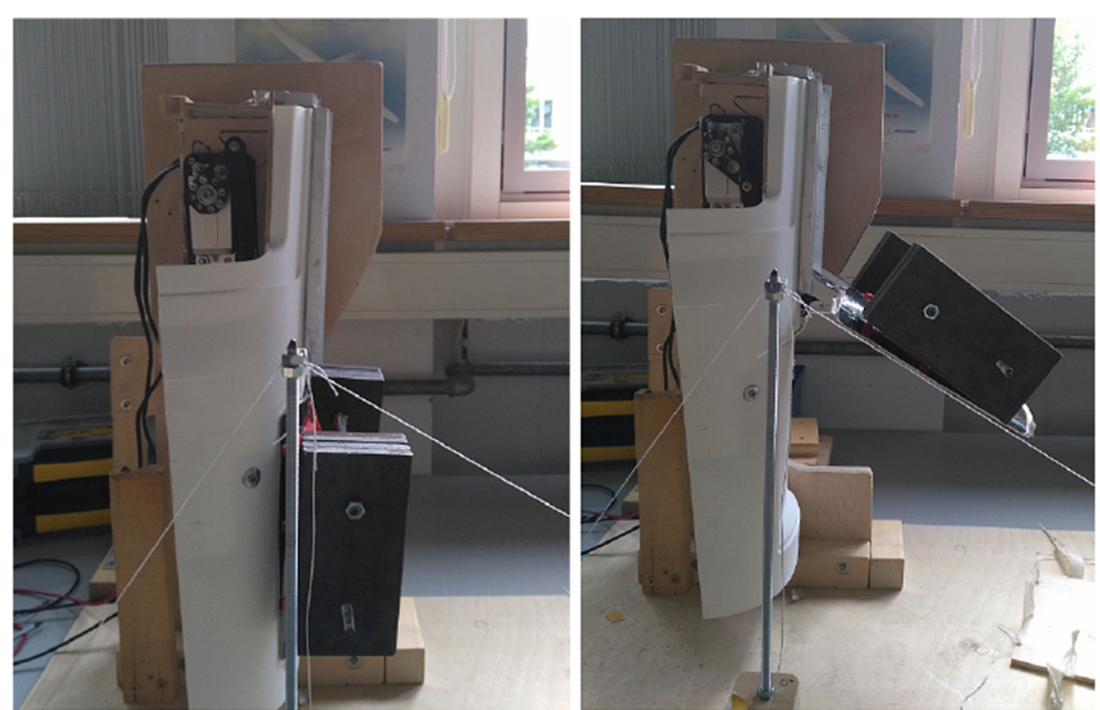
Fig. 9 Servo test bench without load (5: UAV actuator power supply \& command, 6: optical encoder, the source is [4])
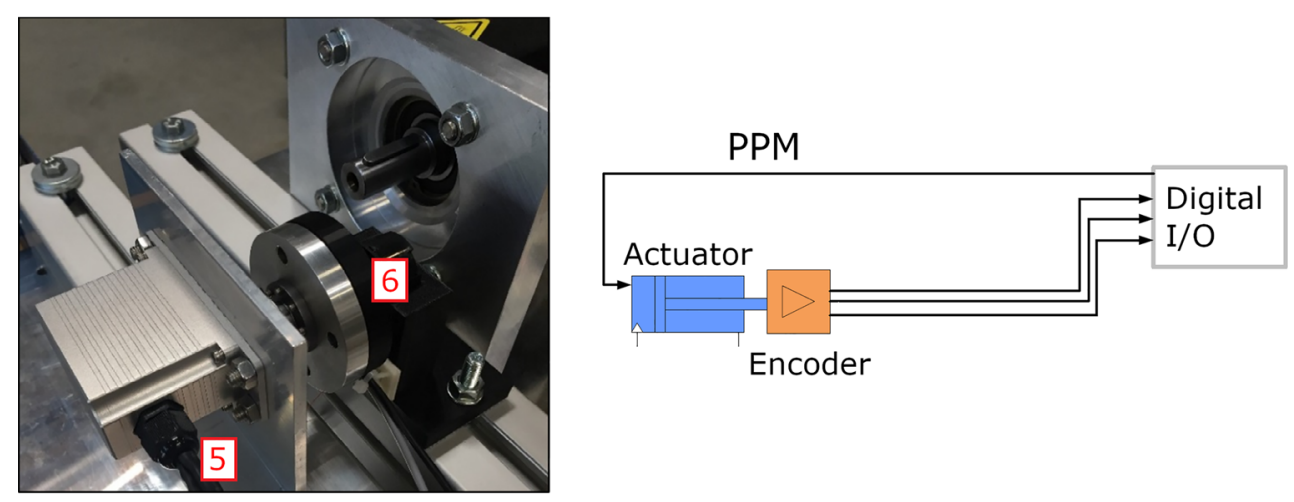

convenience, that classical model-based control algorithms can be applied on them. However, in the present case, several nonlinearities are present in the servo actuator e.g.: the built-in angular position controller with current and therefore angular velocity limitation and friction in the airbrake actuator gearbox which dissipates kinetic energy nonlinearly. For this reason a simple linear model representation of the airbrake actuator dynamics should be avoided. The implemented test scenarios are therefore tailored to the actual operation profile of the airbrake, which is designed to deflect against the airstream around the fuselage. Combined with the propulsive engine, it is used to help aircraft deceleration during the mission therefore dynamic deflections are expected in flight. Three test campaigns are conducted with the loaded test bench (Table 4).

The results of the first two campaigns (Test campaign I \& II) and a detailed description of the test setup are found in [4] while results of Test campaign III are newly generated applying gradually increasing load as the servo
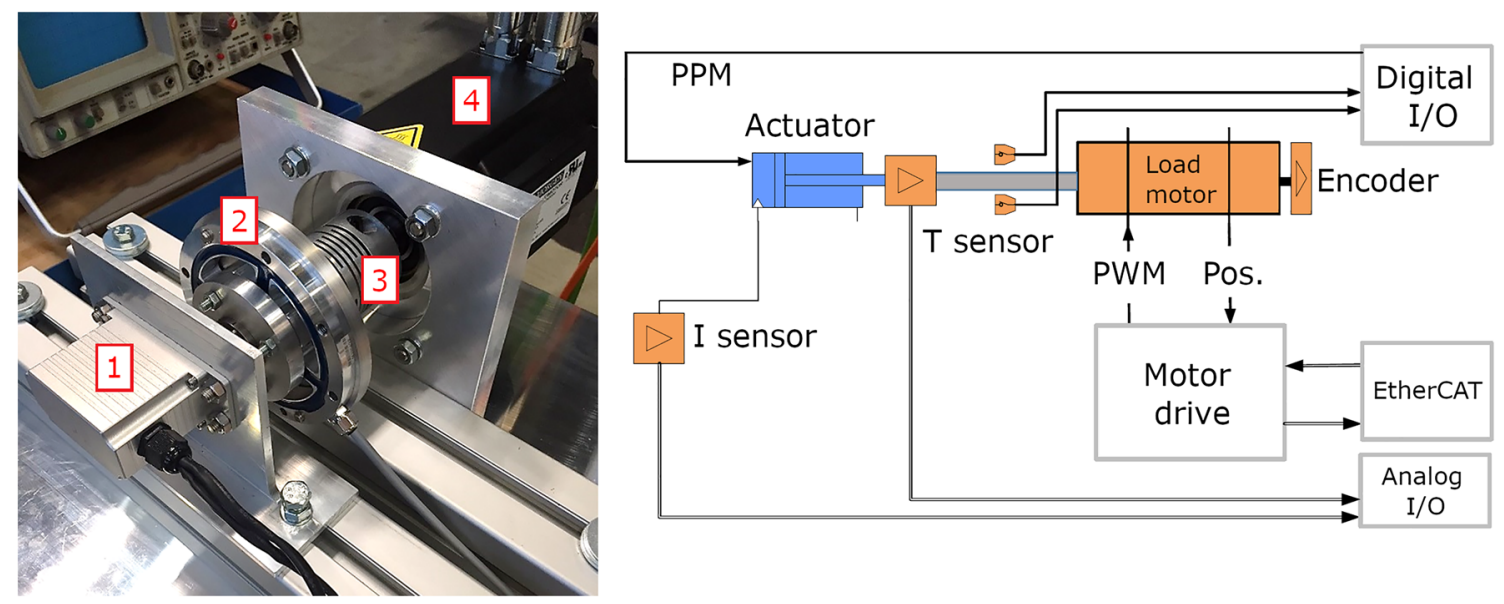

Fig. 10 Servo test bench with load (1: UAV actuator under test, 2: Torque sensor, 3: Beam coupling, 4: Load motor), the source is [4]) 

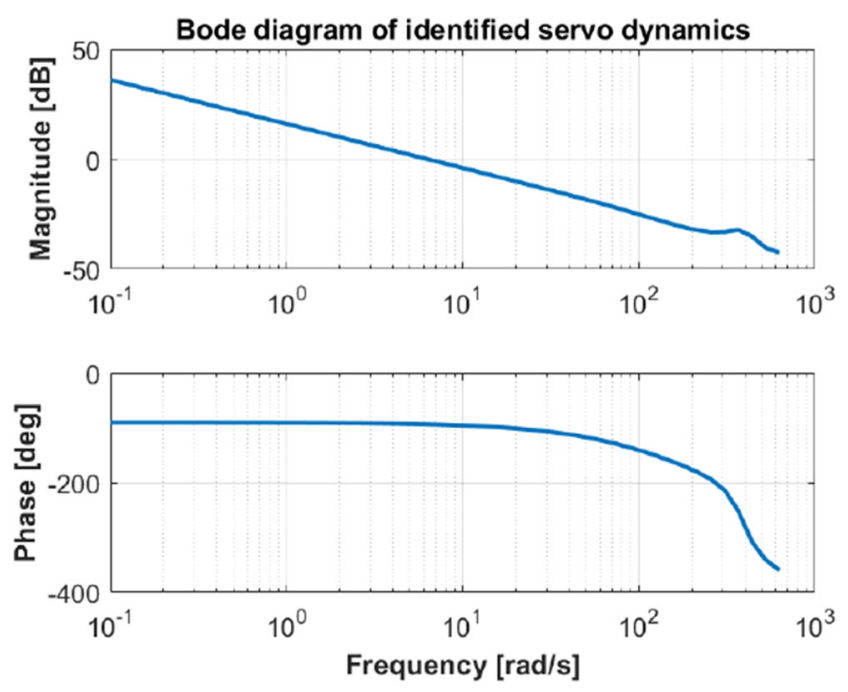

Fig. 11 Bode diagram of servo dynamics

moves and used only in this article. Evaluation of servo actuator performance in this realistic campaign is shown in Table 2 for different loads and the same deflection command $\left(97.5^{\circ}\right)$. Later Test campaign III data is applied to verify the realistic behavior of the servo model.

While the described test bench offered useful experimental data satisfactory to identify the basic mathematical model of the airbrake servo certain limitations are to be pointed out and discussed:

1. Due to the hinge kinematics of the airbrake mechanism, radial forces are exerted against the actuator shaft, in addition to the torsional moment. However, at the test bench the artificial load applied is solely the torsional moment, the radial forces are thus neglected. Depending on the level of the latter, additional friction on the actuator shaft bearing will arise. Successful validation of the identified airbrake model in flight tests (see Section 5.2 and Fig. 55 for example) has shown that there is no significant such effect in the real system.

2. The load torque control algorithm of the load motor in the test bench is based on a feedforward path. Although actual torque is available in real time, addition of a torque feedback control path is avoided, due to instabilities encountered. They are probably attributed to a slight delay in the load motor torque signal and the high stiffness of the load path. As the identified servo model describes the dynamics of the servo motor not the load motor its not possible to examine this effect with the identified model. Another issue is that at the beginning of the actuator movement a peak is visible at the torque profile (see Fig. 31) and also the current profile. This should be caused by the feedforward control experiencing a step change in the reference signal and so commanding a very high input at the first time. This assumption is verified by the behavior for gradually increasing load torque command as there is no such peak in the measured torque as Fig. 34 shows.

3. Sampling is conducted with a frequency of $200 \mathrm{~Hz}$. The maximum variation that can be accurately captured is therefore less than $100 \mathrm{~Hz}$. Current data however suggests that fluctuations of the actual signal might be more rapid. However, as the operating range of the airbrake will be at low frequency (bang-bang control with full opening or closing) the exact representation of very high frequency dynamics is not required. As Fig. 28 shows the servo motor is incapable to make full range deflection even at $5 \mathrm{~Hz}$ without load and limited range deflections (Fig. 29) are also reduced by applying load on the servo. So there is no possibility to drive the servo and so the airbrake with high frequency reference inputs and so the limitation of the represented measured frequency range to $0-100 \mathrm{~Hz}$ is more then acceptable. This is also underlined by the Bode diagram (Fig. 11) of the identified servo motor dynamics (series connection of $G_{r e f}(z)$ reference signal dynamics from Eq. 1 considering the maximum $A_{N}\left(T_{L}\right)=6.1319$ gain if $T_{L}=8 \mathrm{Nm}, G_{\text {sys }}(z)$ servo dynamics and the integrator) also underlines this with $6.2 \mathrm{rad} / \mathrm{s}=$ $0.987 \mathrm{~Hz}$ cut-off frequency and $8.85 \mathrm{rad} / \mathrm{s}=1.4 \mathrm{~Hz}$ bandwitdh. The maximum $A_{N}\left(T_{L}\right)$ gain is larger than the maximum $A_{P}\left(T_{L}\right)$ and the maximum gain gives the maximum bandwidth that's why this is considered.

System identification is discussed in the next sections including reproduction of measurement results of the servo test bench and SIL simulation of the whole nonlinear aircraft dynamics with flight test pattern tracking baseline control without and with airbrake application during

Table 2 Evaluation of the airbrake actuator performance

\begin{tabular}{llll}
\hline Load Nm $/ 97.5^{\circ}$ & Steady angle $\left[{ }^{\circ}\right]$ & Overshoot $R_{o}[\%]$ & Rise time $(10-90 \%)[\mathrm{s}]$ \\
\hline 2 & 95.35 & 0.393 & 0.24 \\
4 & 94.77 & 0.01 & 0.264 \\
6 & 94.03 & 0.02 & 0.292 \\
8 & 93.16 & 0.024 & 0.332 \\
\hline
\end{tabular}


Table 3 Servo angular velocity limits

\begin{tabular}{|c|c|c|c|c|c|}
\hline Load $[\mathrm{Nm}]$ & 0 & 2 & 4 & 6 & 8 \\
\hline Factory opening limit $[\mathrm{deg} / \mathrm{s}]$ & 350 & 315.4 & 280 & 243.7 & 209 \\
\hline Measured opening limit [deg/s] & 340 & 290 & 240 & 180 & 125 \\
\hline Measured closing limit [deg/s] & -340 & -500 & -700 & -800 & -1000 \\
\hline
\end{tabular}

deceleration. Finally, flight testing will be applied for airbrake model verification.

\section{Identification of Airbrake Characteristics}

After the design and construction (in the mock-up test bench see Fig. 8) of the airbrake this section deals with the identification of its dynamics. This is started with the identification of the servo dynamics based-on measurements applying servo test bench with load (see Fig. 10) and the verification of the resulting model comparing model outputs with test bench measurements. This servo model can be considered as the inner core of the whole airbrake model. To construct the whole airbrake model the characteristics of the opening mechanism and the aerodynamic load effects should be determined (based-on [9] but extending and improving the results) and integrated considering also the effect of airbrake inertia and the stiffness of the mechanism on the dynamic behavior.

\subsection{Identification of Servo Dynamics}

The selected rotary servo actuator is the KST X30-12-150 servo (see [16] and [9]). Its factory characteristic can be found in [10] page 3 and is summarized in Table 3.
To identify the servo dynamics the test bench presented briefly in Section 3 and in detail in [4] was applied making measurements with different frequency doublet servo angle setpoint inputs and different loads. The parameters of the whole measurement campaign are summarized in Table 4. In the first column the pulse width modulated (PWM) and angular values $(\alpha)$ of servo deflection references are shown. The second column shows the related airbrake angle deflection $(\phi)$ and the further columns show the applied torque load. Note that the upper opening limit of the airbrake is $60^{\circ}$ so the $67.9^{\circ}$ maximum value is a bit higher. Because the servo motor is tested independently on a separate test bench there is no limitation of this angle. The header lines of the blocks show the test signal frequencies. $1-5 \mathrm{~Hz}$ with $1 \mathrm{~Hz}$ steps (Test campaign I) and $0 \mathrm{~Hz}$ static tests were done (Test campaign II). The third block (Test campaign III) shows a test with step change of the servo reference angle from 0 to 97.5 degrees with continuously changing load to different maximum values as if were in case of inflight opening of the airbrake. Considering Fig. 39 shows that not all measurements are relevant regarding airbrake dynamics as on low opening angles the servo torque is also low. Relevant cases are shown with orange color in the table.

Some illustrative measurement plots are shown in Figs. $12,13,14$ and 15 for the 2100-1800 PWM range (0$30 \mathrm{deg}$ servo angle range) at $1 \mathrm{~Hz}$. Figures 12 and 14 show

Table 4 Servo test measurements

Test campaign I

Frequency $[\mathrm{Hz}]$ :

PWM $[\mu s] / \alpha[\mathrm{deg}]$

$1-5$

$1-5$

1-5

$1-5$

$1-5$

2100-1800 / 0-30

$\phi$ [deg]

2100- $1500 / 0-60$

$0-9.2$

$0-24.2$

$1800-1500 / 30-60$

$9.2-24.2$

1500-1200 / 60-90

$24.2-44$

1200-900 / 90-120

$44-67.9$

Test campaign II

Frequency $[\mathrm{Hz}]$ :

PWM / $\alpha$ [deg]

$1500 / 60$

$\phi[\mathrm{deg}]$

24.2

0

Test campaign III

Frequency $[\mathrm{Hz}]$ :

$\mathrm{PWM} / \alpha$ [deg]

$\phi$ [deg]

2100-1100 / 0-97.5

$0-51.6$

0

$-$

\section{Load $[\mathrm{Nm}]$}

$\begin{array}{lll}4 & 6 & 8\end{array}$

$\begin{array}{lll}4 & 6 & 8\end{array}$

$\begin{array}{lll}4 & 6 & 8\end{array}$

$4 \quad 6$

4

0

2

Load [Nm]

4

0

0

8

0

0

0

0

2
Load [Nm]

4
6

8 


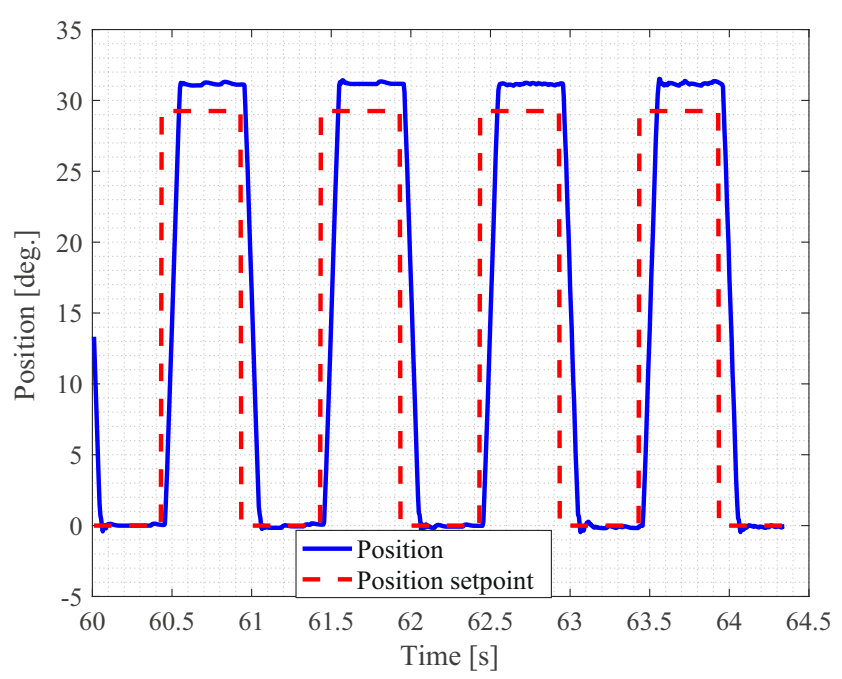

Fig. $121 \mathrm{~Hz}$ servo angle tracking at no load

that the measured servo angle overshoots the reference in case of no load. Note that in Fig. 14 the $8 \mathrm{Nm}$ load is applied between about $64-66.7 \mathrm{sec}$. The overshoot is possibly caused by the transient of the load motor. As the load motor model is not identified this effect can not be studied in detail. Figure 14 shows that when a load is applied this overshoot transforms to undershoot causing negative deflection angles which are theoretically impossible. This can be caused by a missing I-term in servo control. In the servo model identification this change in the steady-state servo angle will be considered by a transfer function from load torque to angle (see Subsubsection 4.1.3). If real flight test results verify this effect then this is built in to the servo model and is not caused by the test bench. Evaluation of flight test results shows that the servo model including this effect well fits the measured data so this is built in to the servo.

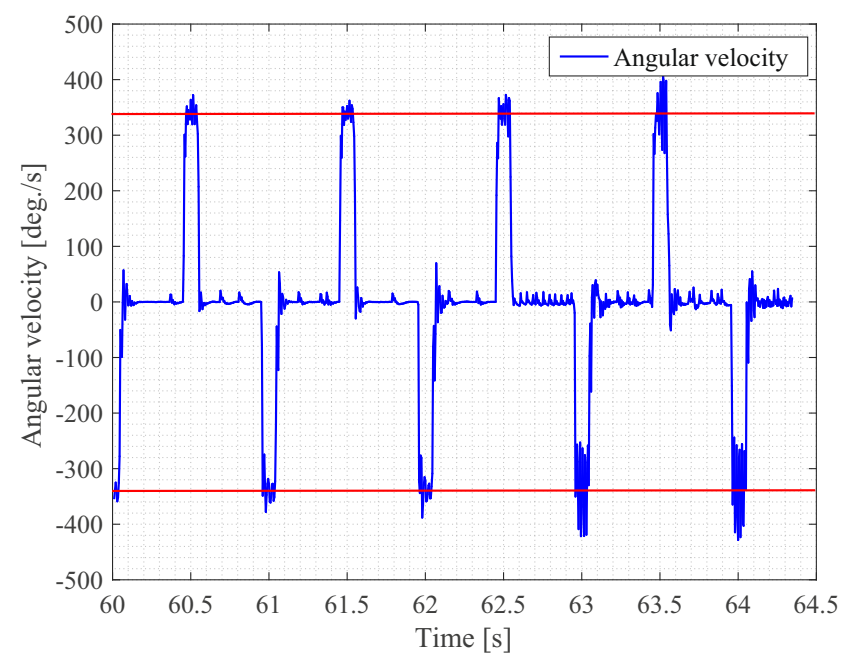

Fig. $131 \mathrm{~Hz}$ angular velocity tracking at no load (Continuous horizontal lines show the steady angular velocity values)

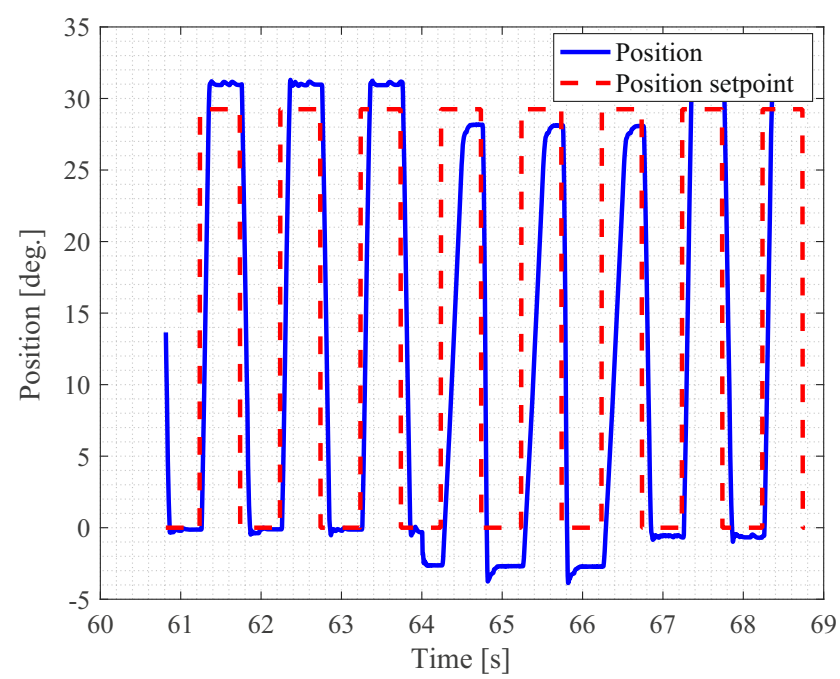

Fig. $141 \mathrm{~Hz}$ servo angle tracking at maximum load $(8 \mathrm{Nm})$

Figures 13 and 15 show that the possible angular velocity is limited inside the servo depending on the load. This is in agreement with the servo datasheet [10] as the first row of Table 3 also shows. However, the real maximum opening velocities against the load are lower then the values from datasheet. The approximate upper bounds are read from the measurements and summarized in Table 3 . In case if the servo moves with the load the angular velocities seem to be almost unlimited, they have much larger absolute minimum values than maximums (except for the no-load case when the min/max angular velocities are the same, see Fig. 13). This case only approximate readings can be done again summarized in Table 3. These are the approximate reference values where the servo seems to converge as in closing

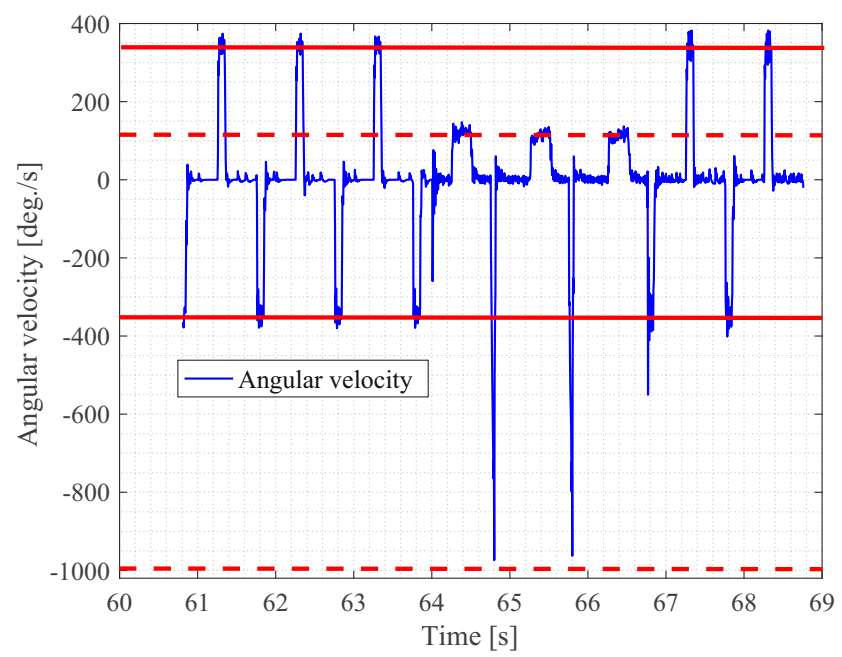

Fig. $151 \mathrm{~Hz}$ angular velocity tracking at maximum load (8Nm) (Continuous horizontal lines show the steady angular velocity values without load, dashed lines show the with / against load values) 
motion the angular velocity is in a transient at all the time (see Fig. 16).

The inner limitation of angular velocity by the actuator electronics made it impossible to identify simple transfer function or state space system models from the reference angle and torque to the output as in [18] for example because there is characteristic difference between opening and closing speeds. That's why nonlinearities were pulledout and identified separately from the transfer function models of the servo. Examining the measured data in detail shows that there can be an inner reference angular velocity value depending on the load and the angle tracking error of the servo. The servo tracks this inner angular velocity reference (see Fig. 15 for example where the maximum angular velocity is almost constant during opening of the airbrake) with a given dynamics. So firstly this inner limitation of the angular velocity reference and its tracking error related dynamics is studied and identified in the next part.

\subsubsection{Transfer Function Identification Between Tracking Error and Angular Velocity Reference}

At first, plotting together the angle tracking error and the angular velocity (for the 1800 to 1500 PWM (30deg to $60 \mathrm{deg}) 1 \mathrm{~Hz}, 0 \mathrm{Nm}$ case in Fig. 17) shows that the angular velocity follows a square reference signal while the tracking error continuously changes. This means that the tracking error should be saturated to get a limited inner angular velocity reference from it. Note that considering Figs. 12 and 14 there is a delay, an offset and a scale error between the reference servo angle and the real one. As possible negative angular values are present in the

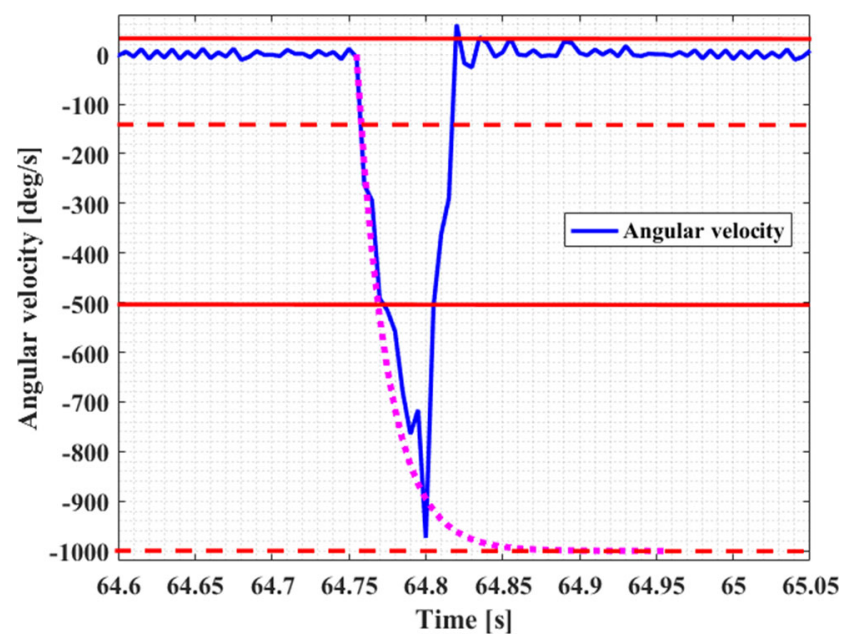

Fig. $161 \mathrm{~Hz}$ angular velocity tracking at maximum load $(8 \mathrm{Nm})$. Dashed lines show the with / against load values. Dotted line shows the exponential type convergence of the angular velocity to the selected minimum value

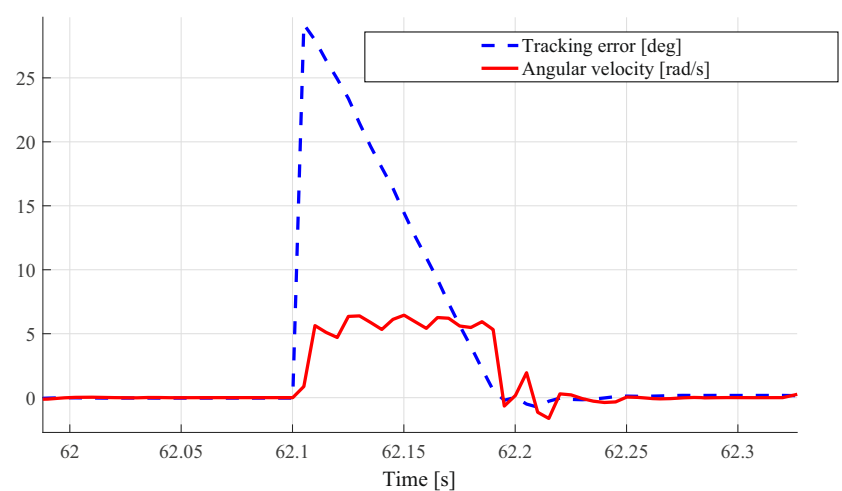

Fig. 17 Tracking error and angular velocity

measurement setup the $30 \mathrm{deg}$ to $60 \mathrm{deg}$ range was selected for identification to avoid them. All of the errors (delay, offset, scale error) were corrected before calculating the tracking error. The delay from the angle reference to the angular velocity was estimated to be 4 time steps (the sampling time is $\Delta t=0.005 s$ ) that's why considering the about one step delay of the discrete time form of transfer functions in Eq. 13 steps delay was applied to the reference signal. The offset and scale error are only considered in the pre-scaling of data for system identification, they are not considered in the final model as they are caused by measurement errors in the test bench.

Studying several cases and considering 3-4 steps delay from the start of saturated tracking error decrease to the start of real angular velocity decrease has shown that the saturation limit of the tracking error should be about $5.8^{\circ}=$ $0.10123 \mathrm{rad}$.

An almost square reference can be easily generated by a simple transfer function from normalized saturated tracking error to angular velocity reference in the form: $G(s)=$ $\frac{A}{T s+1}$ however, for a positive tracking error the velocity reference limit should be different than for a negative and this difference should depend on the load torque $\left(T_{L}\right)$ of the servo. The positive-negative difference can advantageously be described by the following system structure:

$\dot{\alpha}_{r e f}(s)=-\frac{A_{P}\left(T_{L}\right)}{T s+1} \cdot \Delta_{a} \alpha(s)+\frac{A_{N}\left(T_{L}\right)}{T s+1} \cdot \Delta \alpha(s)$

where $A_{P}\left(T_{L}\right), A_{N}\left(T_{L}\right)$ are load torque $\left(T_{L}\right)$ dependent scalar coefficients, $T=0.003 \mathrm{~s}$ is the time constant determined by trial and error to give a realistic reference considering the system answer (see Fig. 18), $\Delta \alpha=\alpha_{\text {ref }}-\alpha$ and $\Delta_{a} \alpha=\left|\alpha_{\text {ref }}-\alpha\right|$. Compared to [9] this model unifies the decision about the opening or closing and the upper and lower angular velocity limitations. In the referenced work separate upper and lower limiting curves were determined, the lower only valid until $2 \mathrm{~Hz}$, here the curves are unified and valid on the whole measured frequency range $(1-5 \mathrm{~Hz})$. This improvement is achieved through the better estimation 


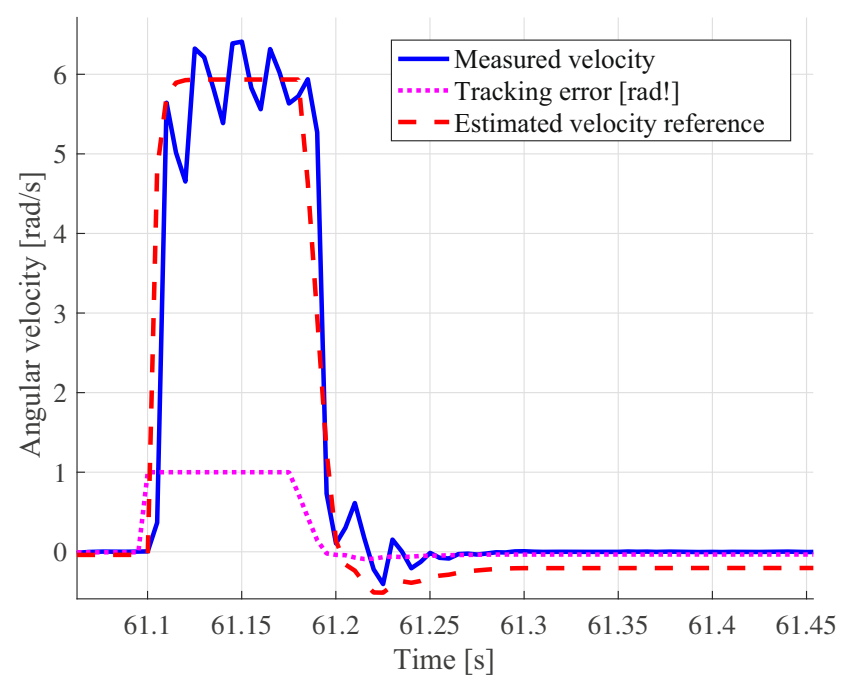

Fig. 18 Identified reference signal dynamics

of closing angular velocities where the attempt was to estimate the reference value to which the servo converges instead of the minimum actual value.

At first, the upper and lower angular velocity limits should be described by possibly linear expressions depending on the load torque then $A_{P}\left(T_{L}\right)$ and $A_{N}\left(T_{L}\right)$ can be determined from this and Eq. 1. This can be satisfactorily done as Figs. 19 and 20 show. The resulted lines (UP and DOWN model in the figures) are:

$\dot{\alpha}_{U P}[\mathrm{deg} / \mathrm{s}]=340-26.5 T_{L}[\mathrm{Nm}]$

$\dot{\alpha}_{D O W N}[\mathrm{deg} / \mathrm{s}]=-340-81.667 T_{L}[\mathrm{Nm}]$

Considering the signs of $\Delta_{a} \alpha$ and $\Delta \alpha$ in cases of upward and downward servo movement needs the following relations can be derived based on Eq. 1:

$\dot{\alpha}_{U P}[\mathrm{deg} / \mathrm{s}]=-A_{P}\left(T_{L}\right)+A_{N}\left(T_{L}\right)$

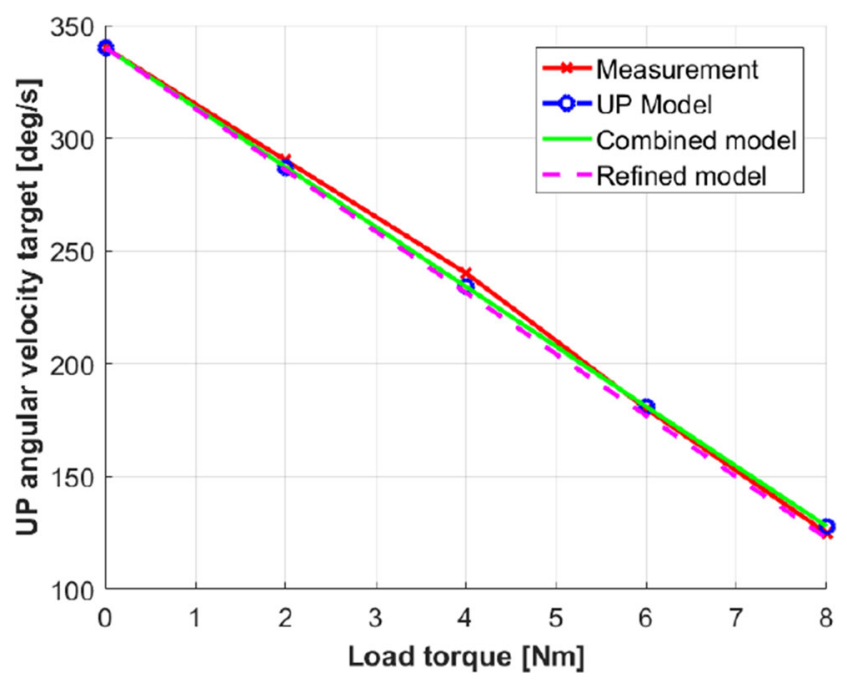

Fig. 19 Approximation of upper angular velocity limit

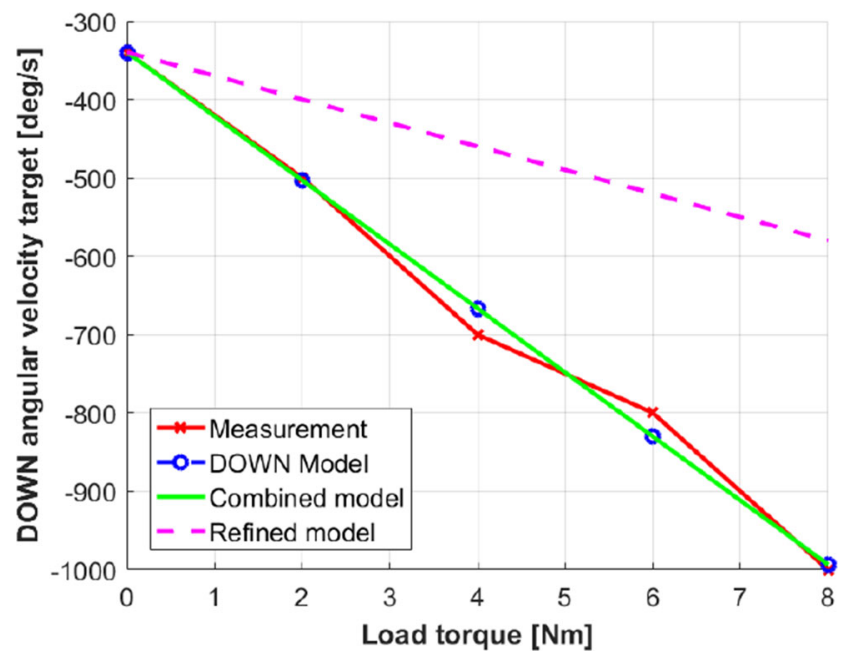

Fig. 20 Approximation of lower angular velocity limit

$\dot{\alpha}_{D O W N}[\mathrm{deg} / \mathrm{s}]=-A_{P}\left(T_{L}\right)-A_{N}\left(T_{L}\right)$

From these expressions its easy to get the transfer function gains in the form:

$A_{P}\left(T_{L}\right)[\mathrm{deg} / \mathrm{s}]=54.0835 T_{L}[\mathrm{Nm}]$

$A_{N}\left(T_{L}\right)[\mathrm{deg} / \mathrm{s}]=340+27.5835 T_{L}[\mathrm{Nm}]$

The above combinations of these gains are also plotted in Figs. 19 and 20 as 'Combined model' and perfectly cover the UP/DOWN curves. Later analysis of the identified servo model (see Figs. 26 and 27) has led to refinement of these coefficients decreasing the upper and increasing the lower limit. The final formulae resulted as:

$A_{P}\left(T_{L}\right)[\mathrm{deg} / \mathrm{s}]=28.5835 T_{L}[\mathrm{Nm}]$

$A_{N}\left(T_{L}\right)[\mathrm{deg} / \mathrm{s}]=340+1.4165 T_{L}[\mathrm{Nm}]$

The curves related to these values are also plotted in the Figs. 19 and 20 ('Refined model'). Of course the formulae have to be converted to give $\mathrm{rad} / \mathrm{s}$ output in the form:

$A_{P}\left(T_{L}\right)[\mathrm{rad} / \mathrm{s}]=0.4989 T_{L}[\mathrm{Nm}]$

$A_{N}\left(T_{L}\right)[\mathrm{rad} / \mathrm{s}]=5.9341+0.02472 T_{L}[\mathrm{Nm}]$

After giving a method to generate inner angular velocity references from angle tracking error and load torque the tracking dynamics of this reference should be identified.

\subsubsection{Servo Dynamics Identification Between Angular Velocity Reference and Angular Velocity}

The servo dynamics from delayed inner angular velocity reference to measured angular velocity was identified using the Matlab System Identification Toolbox output error function considering zero delay and varying numerator and denominator polynomial degrees in discrete time. Finally, zero numerator and 3 denominator degrees (similarly to [8]) 


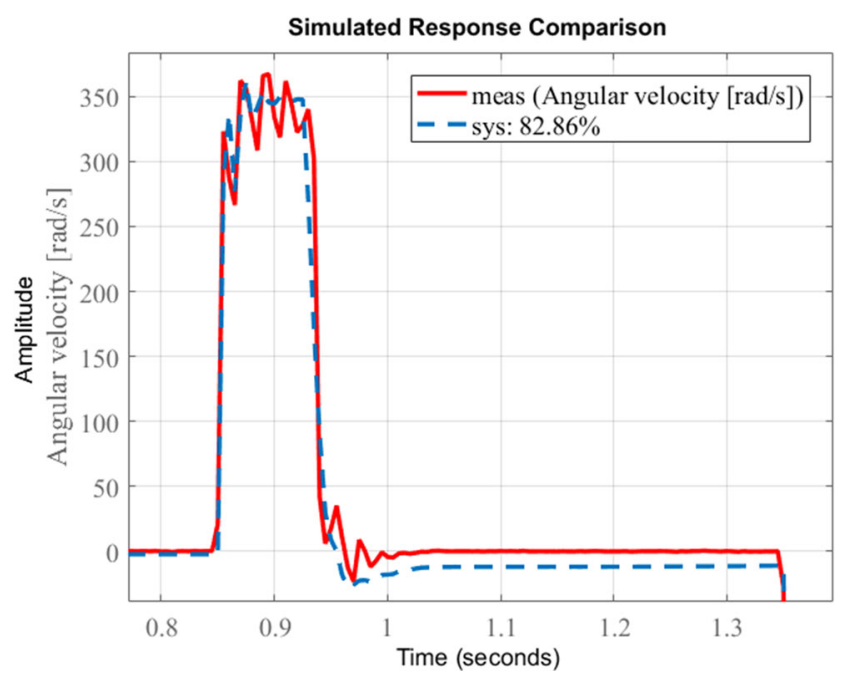

Fig. 21 Identified servo system dynamics

gave the best results as shown in Fig. 21 with $82.86 \%$ fit. This transfer function $\left(G_{s y s}(z)\right)$ has no zeros and poles:

\section{$0.4882-0.2515 \pm 0.6483 i$}

These discrete time poles are stable and oscillatory as the measured system output is also oscillatory. The parameters of the transfer function are shown in the Appendix. The slight offset error in the figure is the result of non-perfect offset removal from reference - output signal pair (see Fig. 18 where the estimated velocity reference is only approximately zero). Integrating the real angular velocity of the servo model gives servo deflection angle so this way the identification of the servo dynamics is ready. However, a load dependent angular position offset was detected in the test bench measurements (see Fig. 14) which should be also identified. This is done in the next part.

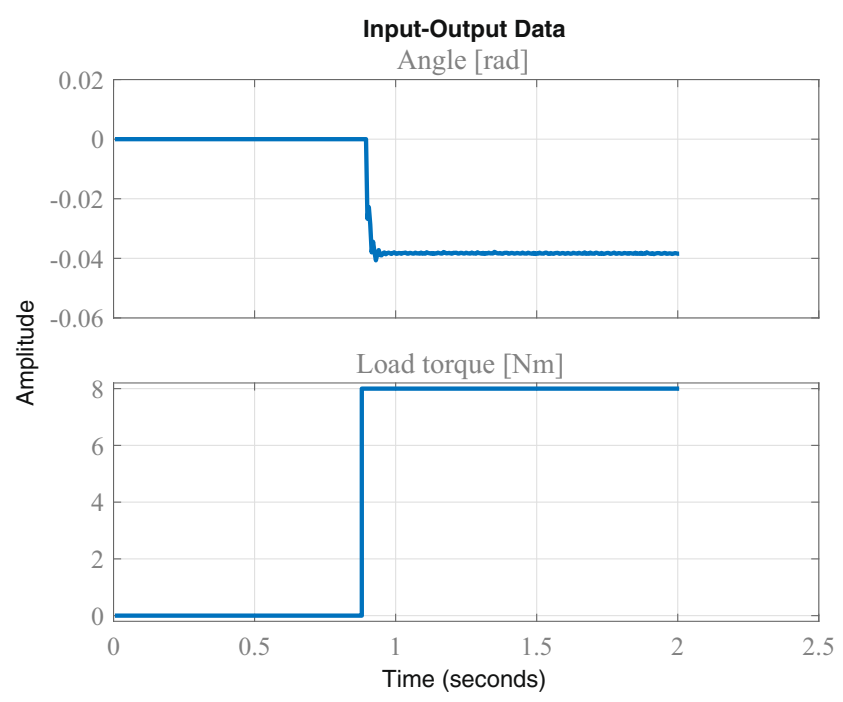

Fig. 22 Effect of load application on servo angle

\subsubsection{Load Dependent Servo Angle Offset Dynamics}

Examining the measured data shows that application of the load moves the system steady-state servo angle with some value. This can be caused by a missing I-term in servo control electronics. Identification of the load torque $\left(T_{L}\right)$ output angle $(\alpha)$ offset dynamics can be done considering load application in the fix 1500 PWM measurement cases. The delay between load and angle was estimated as 4 steps and then a transfer function $\left(G_{T_{L}}(z)\right)$ with zero numerator and one denominator degree was identified with $96.98 \%$ fit (parameters published in Appendix). The load change and angle change are shown in Fig. 22 and the transient of the identified transfer function is shown in Fig. 23.

After identifying the reference and system dynamics a Matlab Simulink simulation for the servo model itself was constructed and the identified model verified driven by test bench data with load application. The block scheme of the Simulink model is shown in Fig. 24.

The block delay represents the 3 steps time delay of the reference. $S A T$ and NORM represent saturation of the error signal to $\pm 0.10123 \mathrm{rad}$ and normalization with $\frac{1}{0.10123}=9.8786$ respectively. $G_{\text {ref }}(z)$ represents the discrete time equivalent of the identified inner angular velocity reference model (1) $G_{s y s}(z)$ is the identified system model from angular velocity reference to angular velocity and $G_{T_{L}}(z)$ is the identified system model from load to angle including also the 4 steps time delay.

\subsubsection{Servo Model Verification Based-on Measured Test Bench Data}

After the identification and Simulink model construction the dynamic model of the servo motor was verified basedon measured data from the servo test bench. As a first

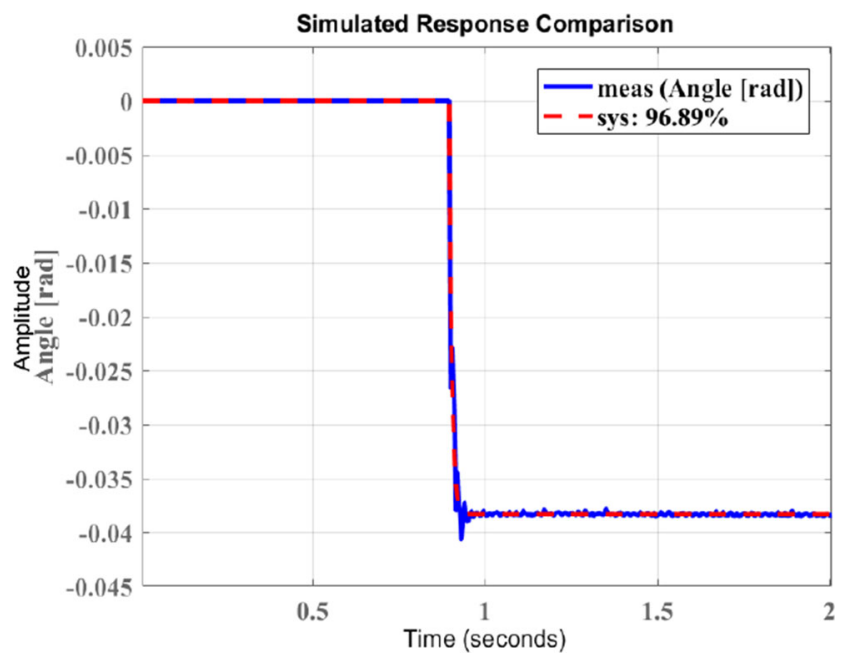

Fig. 23 Transient of identified load to angle system model 
Fig. 24 Simulink model of the servo

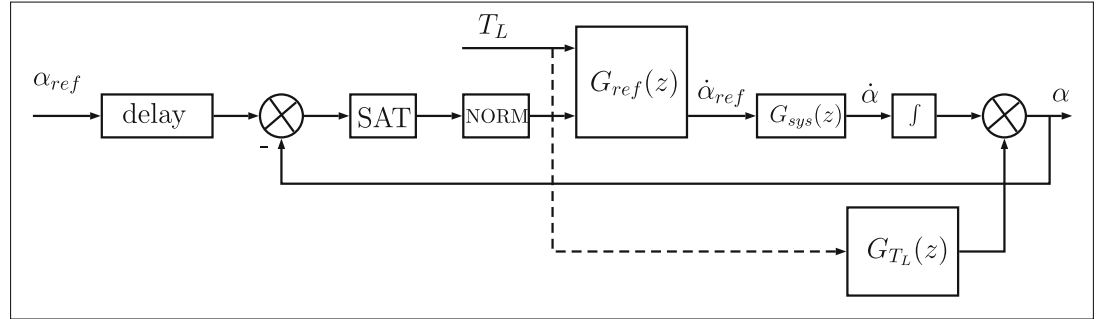

verification simulation results of the 2100 to $1800 \mathrm{PWM}$, $1 \mathrm{~Hz}, 0 \mathrm{Nm}$ and $4 \mathrm{Nm}$ measurement cases are shown in Figs. 25 and 26. $4 \mathrm{Nm}$ is the maximum realistic load in this deflection range that's why it was considered in model verification instead of the $8 \mathrm{Nm}$ maximum load. Results of a simulation with the original lower angular velocity reference bounds with 4Nm load are also shown in Fig. 27. The first two figures show that the final system model acceptably models the servo dynamics both without and with load. In the third figure it can be seen that the original lower angular velocity bounds gave very large overshoot in case of downward moving servo that's why the limits were increased (decreased in absolute value) and the expressions for $A_{P}\left(T_{L}\right)$ and $A_{N}\left(T_{L}\right)$ were modified.

As Fig. 24 shows the required inputs of the servo model are reference deflection and load torque. The reference deflection is given for all test bench measurement (see Fig. 26 for example) and the real load torque of the servo is saved (see for example Fig. 31) so both can be given as simulation model input.

At first, the identified model was checked considering a measurement from Test campaign $I$ with full range deflection $0-120^{\circ}$ at $5 \mathrm{~Hz}$ square wave excitation with $8 \mathrm{Nm}$ load application through some time. This is the most critical test case with the highest frequency and load. As Fig. 28

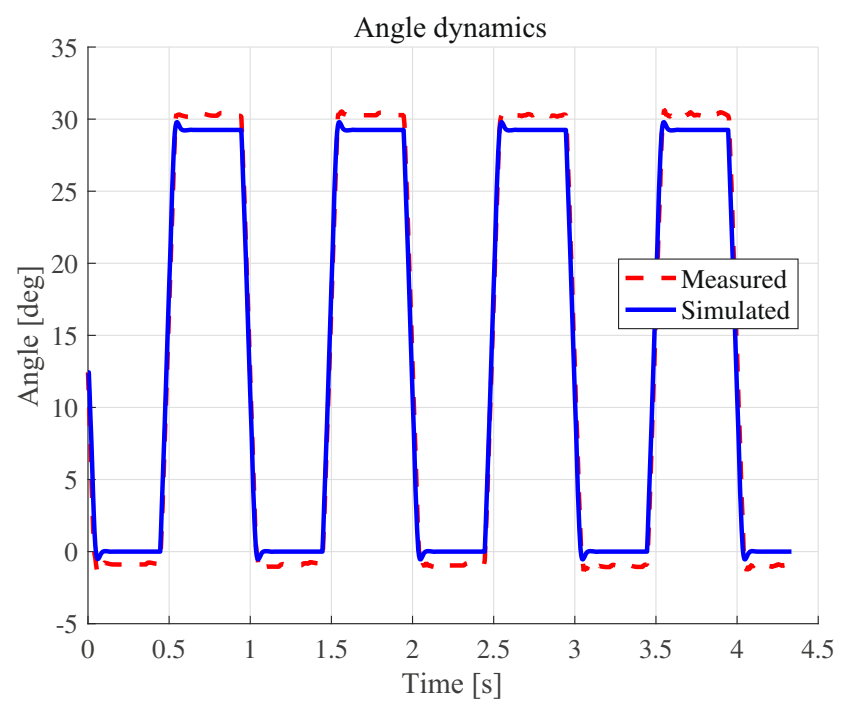

Fig. 25 Servo model with 0 to 30 degs reference zero load shows this range with this frequency is too challenging for the servo controller and it can not follow the reference signal even with zero load. Comparison with data from the identified model shows that there is a drift in the test bench behavior which is not considered in identification.

Checking the model for test bench measurements with a tractable range $\left(30-60^{\circ}\right)$ again from Test campaign I in Fig. 29 shows that in normal mode there is neither drift in test bench output nor in identified model. Figure 29 also shows that the identified model well follows the behavior of the real servo considering the angular deflections. Figure 30 shows the measured and simulated angular velocities and Fig. 31 shows the reference and the measured torque of the test bench for completeness.

The simulated angular velocities are close to the real ones except for the transient periods but the overall performance is acceptable. So finally, the largest difference between model and measurements is the downward overshoot of the angle and the different behavior in the transients (when torque is applied and removed). However, in these test bench measurements the maximum load torque was applied immediately which is not realistic considering the opening of the airbrake, there the maximum load torque appears only on maximum deflection and it increases gradually as the

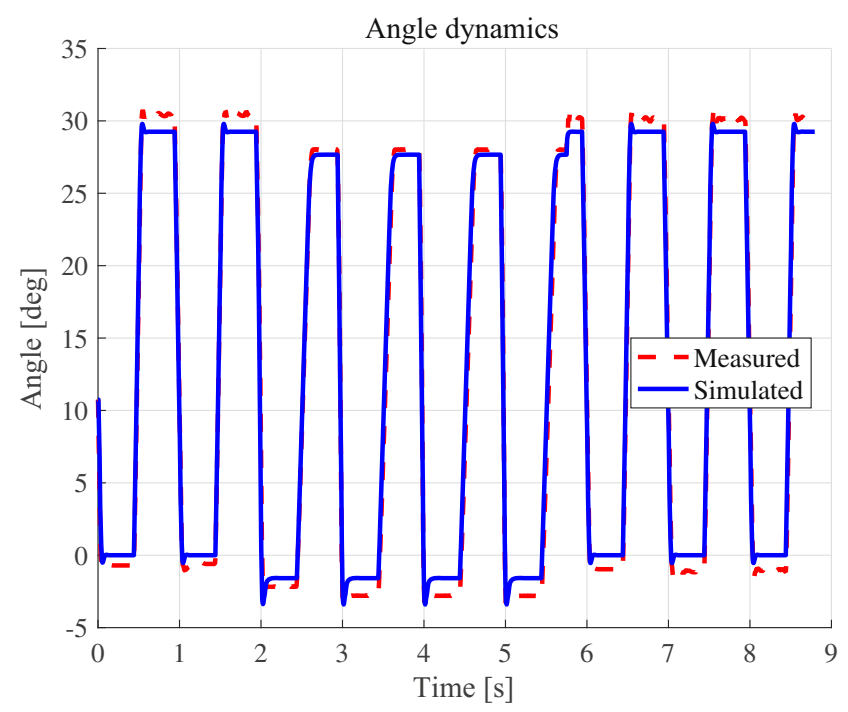

Fig. 26 Servo model with 0 to $30 \mathrm{degs}$ reference $4 \mathrm{Nm}$ load tight angular velocity limit 


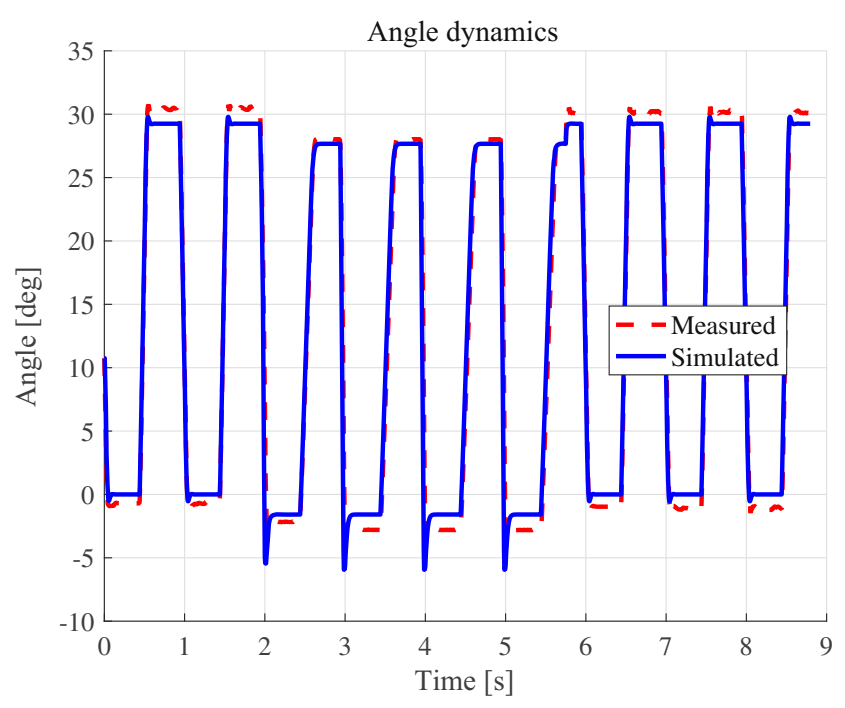

Fig. 27 Servo model with 0 to 30 degs reference $4 \mathrm{Nm}$ load loose angular velocity limit

airbrake is opened. So if the model works well for gradually increasing realistic load torque then the different transients in unrealistic circumstances do not cause a problem.

To verify the servo model with more realistic conditions test bench measurements with gradually increasing (following the increase in opening angle) load torque from Test campaign III are considered. Figures 32, 33 and 34 show the results for the maximum load case when servo deflection to $97.5^{\circ}$ is followed by a load increase to $8 \mathrm{Nm}$. The figures show that both the simulated angular velocity and angle follow well the measurements so the description of the real dynamics is satisfactory. The only difference is a large angular velocity glitch in the measurements when the

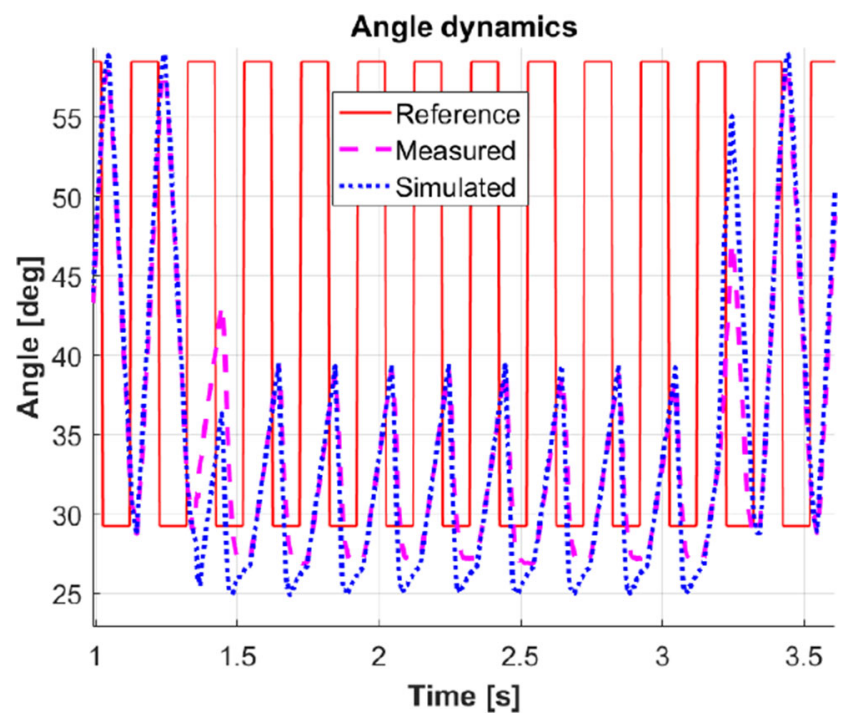

Fig. 29 Measured and simulated servo angle dynamics in limited range $\left(30-60^{\circ}\right)$ at $5 \mathrm{~Hz}$ and $8 \mathrm{Nm}$ square load

load is suddenly removed (see Fig. 34). Carefully examining Fig. 32 shows that the identified model well follows the small angle change in this case (at about 12.5s) and so the lack of the glitch does not cause any discrepancy and so the identified model's behavior is acceptable.

After the verification of the servo model the static characteristics of the opening mechanism and the airbrake aerodynamic effects are examined and described in the next subsection together with the possible dynamic effects of opening mechanism stiffness and airbrake inertia.

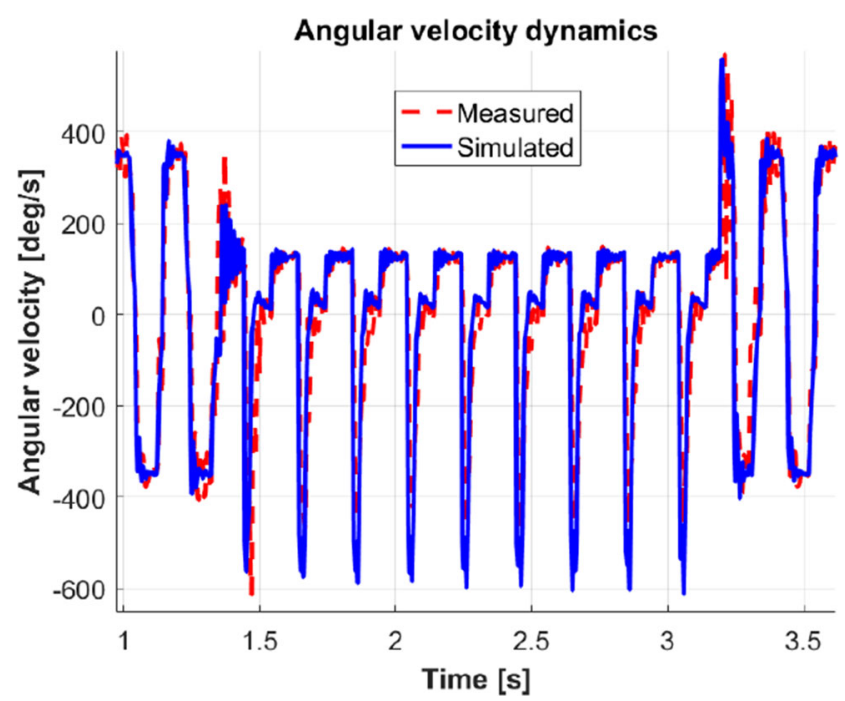

Fig. 30 Measured and simulated servo angular velocity dynamics in limited range $\left(30-60^{\circ}\right)$ at $5 \mathrm{~Hz}$ and $8 \mathrm{Nm}$ square load
Fig. 28 Measured and simulated servo angle dynamics in full range

$\left(0-120^{\circ}\right)$ at $5 \mathrm{~Hz}$ and $8 \mathrm{Nm}$ square load

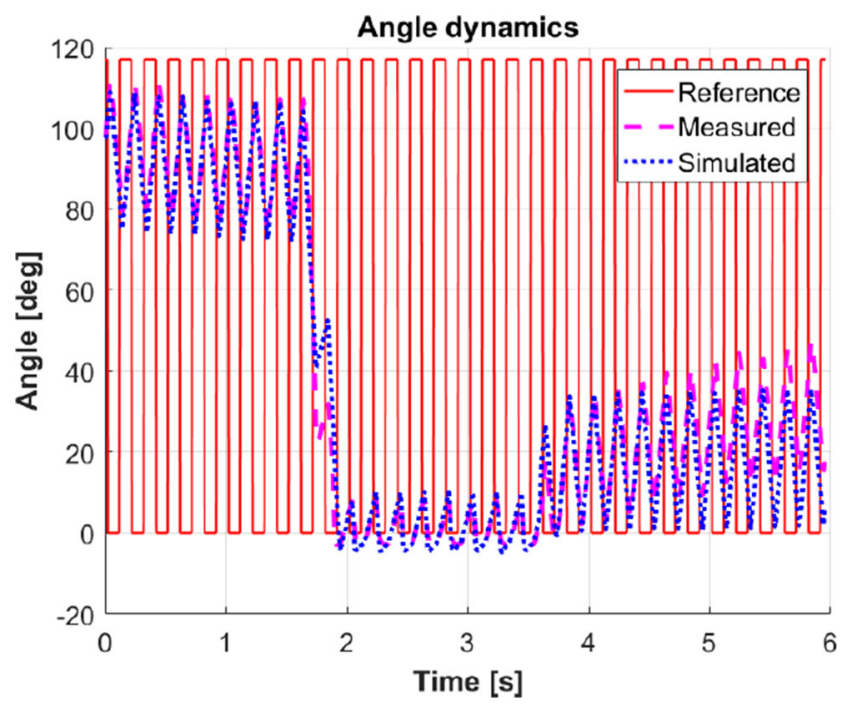




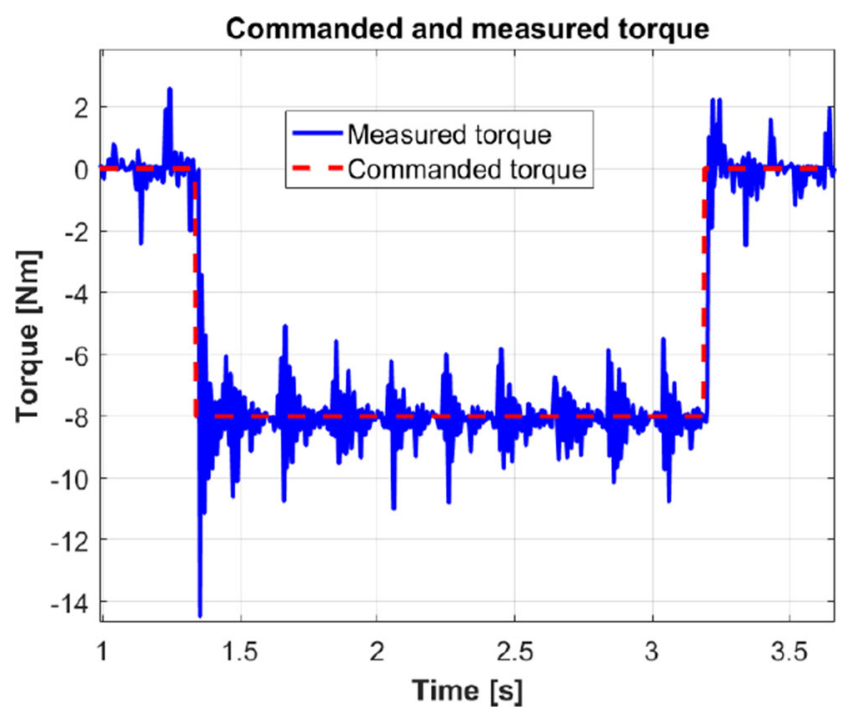

Fig. 31 Referenced and measured servo load torque (8Nm square load) in limited range $\left(30-60^{\circ}\right) 5 \mathrm{~Hz}$ experiment

\subsection{Determination of Static Characteristics}

The most important static characteristic of the airbrake is the aerodynamics. Considering the dynamic pressure $p_{d y n}=$ $\frac{\rho}{2} V^{2}$ known (here $\rho$ is air density and $V$ is airspeed) the aerodynamics can be described by the drag force $c_{D}$ and normal force $c_{N}$ coefficients depending on the opening angle $\phi$ of the airbrake. The normal force coefficients are published in [9] while the drag force coefficients are first published here in Table 5.

Regarding the drag the calculations were done considering a flat plate with a given opening angle based on [12]. Plotting the data together (see Fig. 35) it seems to be

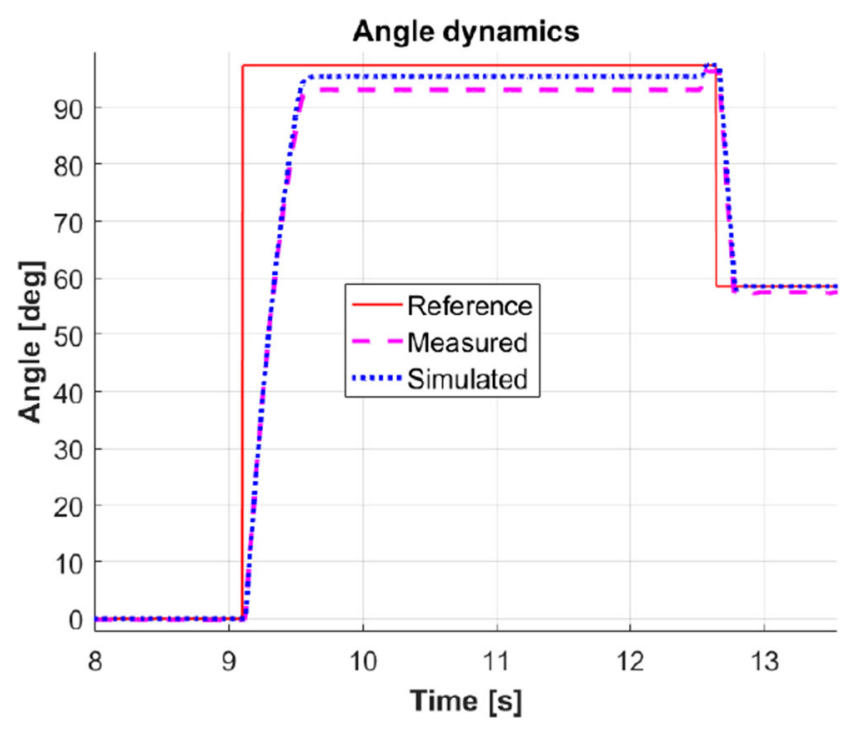

Fig. 32 Measured and simulated servo angle dynamics with gradually increasing load

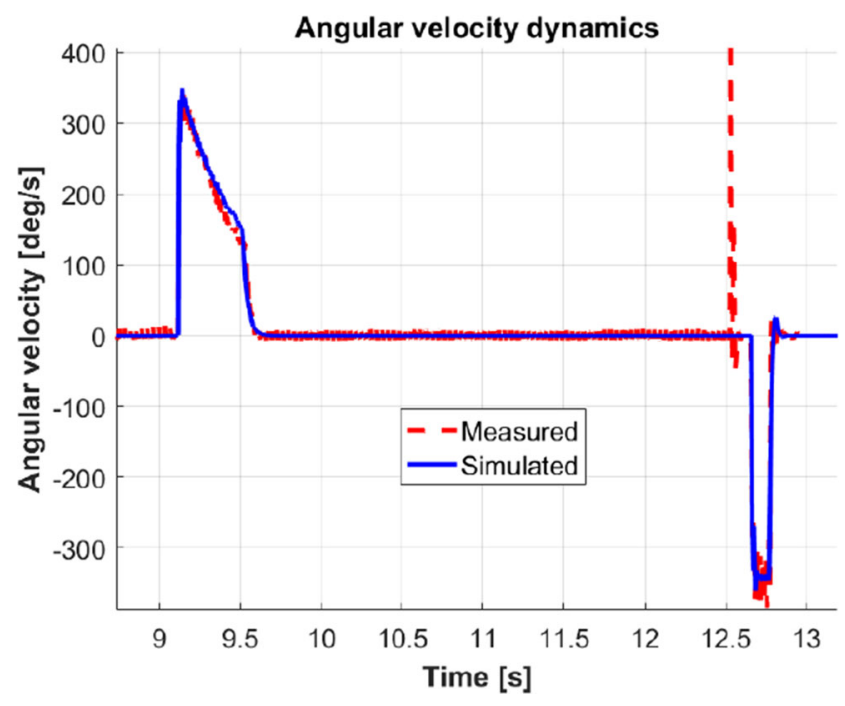

Fig. 33 Measured and simulated servo anglular velocity dynamics with gradually increasing load

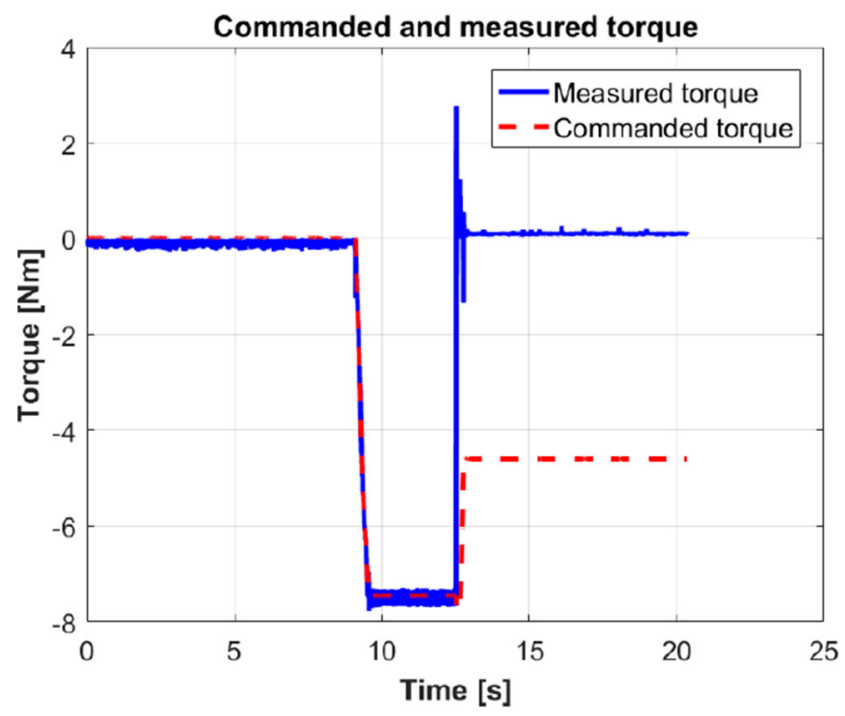

Fig. 34 Gradually increasing load in servo test bench

Table 5 Aerodynamic data

\begin{tabular}{lllll}
\hline$\phi\left[^{\circ}\right]$ & $c_{N}$ & $a[\mathrm{~mm}]$ & $c_{N}^{\prime}$ & $c_{D}$ \\
\hline 0 & 0 & 106.058 & 0 & 0 \\
10 & 0.3 & 118.985 & 0.3366 & 0.18 \\
20 & 0.65 & 131.912 & 0.8085 & 0.3 \\
30 & 0.9 & 144.839 & 1.2291 & 0.5 \\
40 & 1.21 & 157.766 & 1.8 & 0.8 \\
50 & 1.1 & 170.693 & 1.7704 & 0.75 \\
60 & 1.05 & 183.62 & 1.8179 & 0.9 \\
\hline
\end{tabular}




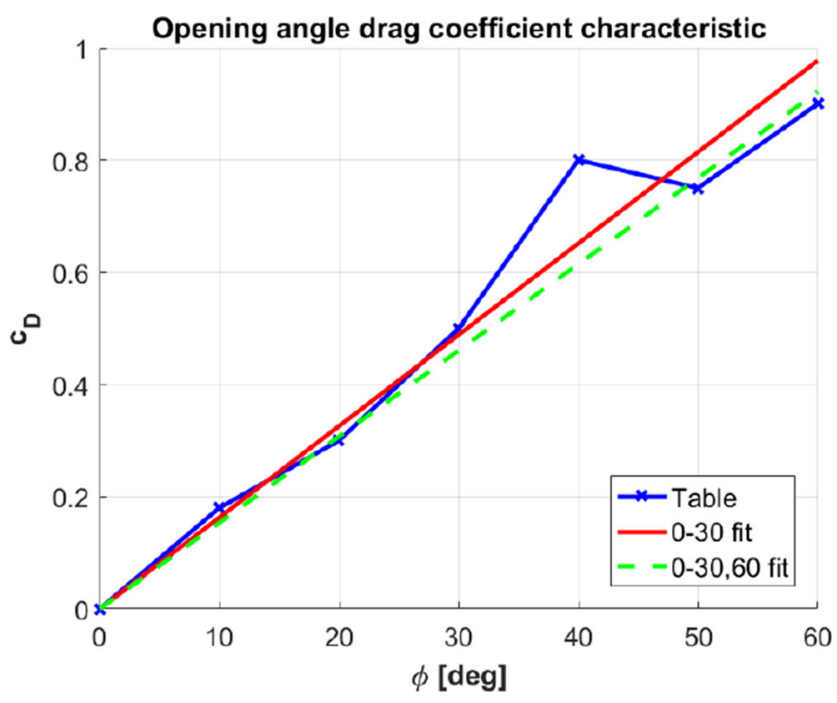

Fig. 35 Line fit to airbrake angle - drag coefficient data

that the value at $40^{\circ}$ is an outlier as it is out of the otherwise linear trend and so it is neglected when fitting a line to the data. A constrained line fit going through the $\left(0^{\circ}, 0\right)$ point was done considering only opening angles $\left[\begin{array}{lllll}0^{\circ} & 10^{\circ} & 20^{\circ} & 30^{\circ} & 60^{\circ}\end{array}\right]$ to provide a more balanced fit with two points below and two above the resulting line (see Fig. 35). Considering also the $50^{\circ}$ point moved the fit down almost neglecting the upper points. On the contrary considering only the $0^{\circ}$ to $30^{\circ}$ range gave a line too up almost neglecting the lower points as the figure shows. The resulting polynomial $c_{D}(\phi)$ can be found in the Appendix.

Regarding the normal force coefficient its application point also changes with the opening angle. This is characterized by the $a(\phi)$ arm length in Table 5. As the final goal is to get an airbrake model as compact as possible its worth to unite the normal force coefficient and the arm length as

$c_{N}^{\prime}(\phi)=c_{N}(\phi) \frac{a(\phi)}{a_{\text {ref }}}$

where $a_{\text {ref }}=106.058 \mathrm{~mm}$ is the assumed fixed arm length. Considering the normalized $c_{N}^{\prime}(\phi)$ coefficient two sections of the curve can be distinguished and so a parabola and a line were fitted to the data as shown in Fig. 36. The formulae are presented in the Appendix. Based on $c_{N}^{\prime}(\phi)$ the normalized normal force can be calculated as $F_{N}^{\prime}=c_{N}^{\prime}(\phi) p_{d y n} S_{r e f}$ where $S_{r e f}=0.032675282 m^{2}$ is the airbrake reference area (on one side).

The required servo torque to move the airbrake can be calculated considering the geometry of the opening

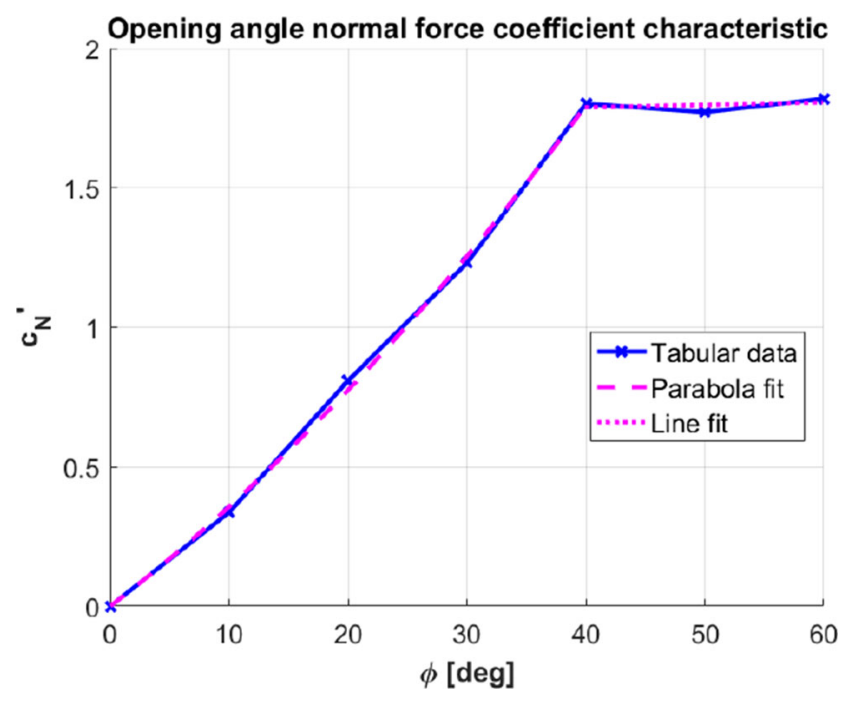

Fig. 36 Curve fit to airbrake angle normal force coefficient data (continuous line: original data, dashed line: fitted curves)

mechanism in between shown in Fig. 37. In the figure the arm lengths are:

$$
\begin{aligned}
a_{r e f} & =106.058 \mathrm{~mm} \\
b & =68.417 \mathrm{~mm} \\
c & =29.829 \mathrm{~mm} \\
d & =112.698 \mathrm{~mm} \\
e & =42.932 \mathrm{~mm}
\end{aligned}
$$

There is a nonlinear relation between the angles $\beta, \gamma, \delta, \phi$ summarized in tabular form in Table 6 . Note that for small $\beta$ angles $\delta$ can be negative as shown in Fig. 37 and for large $\beta$ angles $\gamma$ becomes negative (it is shown in the positive range in the figure).

The servo torque for a given airbrake opening angle $\phi$, airspeed $V$ and air density $\rho$ can be determined as:

$$
\begin{aligned}
T(\phi) & =\frac{e}{1000} \frac{\cos (\delta(\phi))}{\cos (\gamma(\phi))} \frac{a_{r e f}}{b} p_{d y n} S_{r e f} \cdot c_{N}^{\prime}(\phi) \\
& =s_{a}(\phi) c_{N}^{\prime}(\phi) p_{d y n}
\end{aligned}
$$

In the second part of the equation all the geometric parameters are summarized in a virtual servo arm length $s_{a}(\phi)$. The curves for $c_{N}^{\prime}(\phi)$ are determined before so a curve for the $s_{a}(\phi)=\frac{e}{1000} \frac{\cos (\delta(\phi))}{\cos (\gamma(\phi))} \frac{a_{\text {ref }}}{b} S_{\text {ref }}$ virtual servo arm is required. Substituting the known fix parameters the only angle dependent part remains $\frac{\cos (\delta(\phi))}{\cos (\gamma(\phi))}$ :

$s_{a}(\phi)=\frac{42.932}{1000} \frac{106.058}{68.417} 0.032675282 \frac{\cos (\delta(\phi))}{\cos (\gamma(\phi))}=2.1744 \cdot 10^{-3} \frac{\cos (\delta(\phi))}{\cos (\gamma(\phi))}$

The tabular data and the fitted 5th degree curve are shown in Fig. 38. The curve parameters are given in the Appendix.

After fitting polynomial models to the normal force coefficient and the virtual servo arm it is advisable to check 


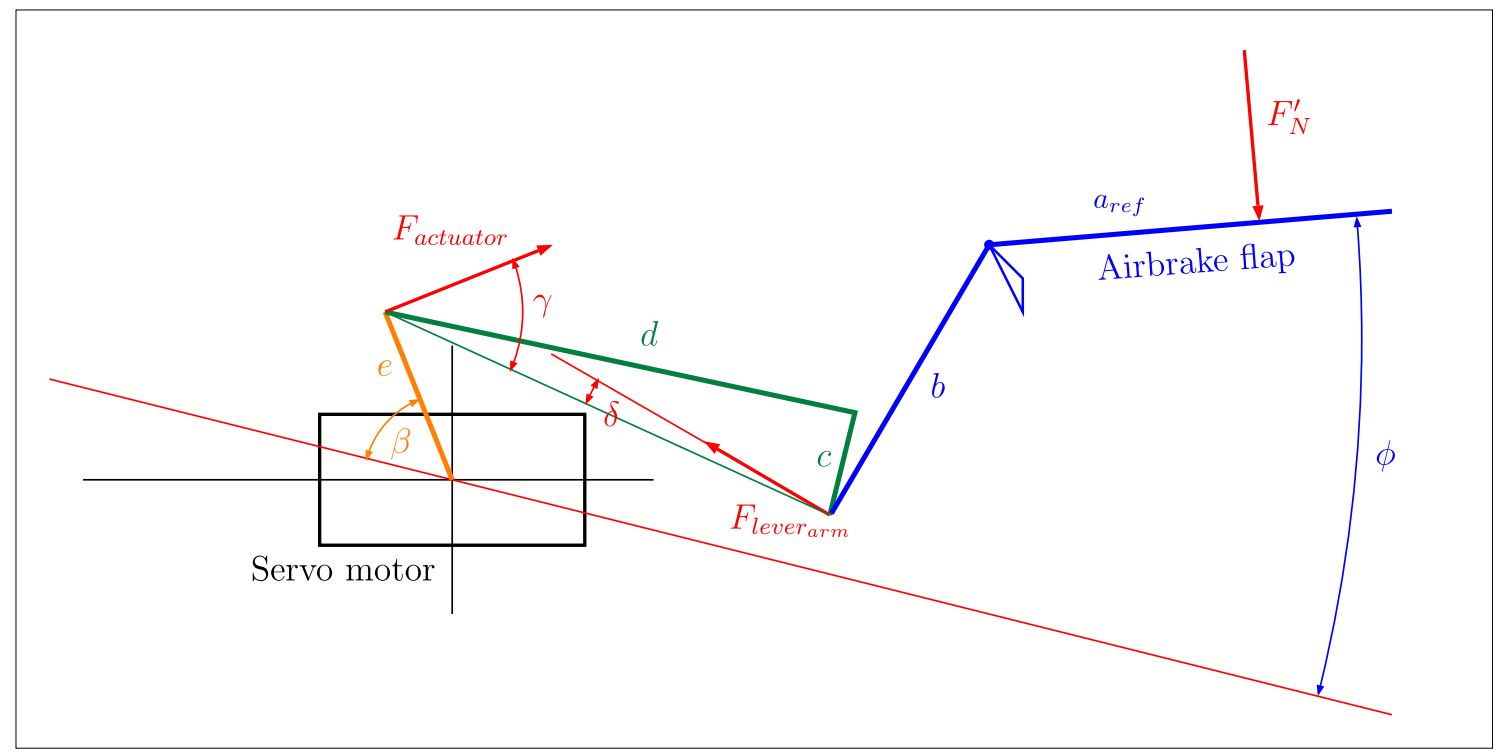

Fig. 37 The opening mechanism of the airbrake ( $\beta$ servo arm angle, $\phi$ airbrake opening angle

the precision of fitting. The largest servo torque will be required on sea level so servo torque values are calculated on sea level for airspeed from $10 \mathrm{~m} / \mathrm{s}$ to $60 \mathrm{~m} / \mathrm{s}$ and for all possible $\phi$ opening angles from Table 5. Calculations were done both from the mechanism and tabular data and from the fitted curves. Figure 39 shows that the approximate (dashed) curves are close to the real ones at every point while the absolute difference increases with airspeed. The relative errors of the fits (percentage) are shown in Fig. 40. These percentages are independent from the air density and airspeed. The figure shows that the maximum error is about $6 \%$ at $\phi=10^{\circ}$ and then the errors are decreasing. The relative error can not be calculated for zero angle with zero torque that's why it is not plotted. Extrapolating the other values the relative error around zero angle can be about $8-10 \%$.

After fitting curves to the aerodynamic coefficients and moment arm fast conversion formulae from servo angle $\alpha$ to airbrake angle $\phi$ and vice versa are required. The servo angle $\alpha$ is different form the servo arm angle $\beta$. It is defined to be zero at the minimum servo arm angle: $\alpha=\beta-18.612^{\circ}$. Regarding the curve fits $\alpha-\phi$ resulted as a 3 rd degree polynomial going through zero while $\phi-\alpha$ as a 4th degree polynomial again going through zero. The polynomials are summarized in the Appendix while the fit qualities can be checked in Fig. 41 (they are superior).

Finally, the stiffness characteristics of the airbrake mechanism should be identified to increase precision of deflection model considering the flexibility of the mechanism. Theoretical airbrake deflection angle from commanded actuator position and transmission of the rigid kinematic chain and measured deflection angle from the airbrake mock-up (see Fig. 8) were compared for a given set of measurement points. From the angle deviation, the system stiffness was derived (for details see [9]). The goal of this work was to extend the covered range by the stiffness formula as only the $\phi$ opening range $20-60^{\circ}$ was covered by the model in [9]. Finally, the stiffness data was converted from degrees $\phi$ angle input and output to radians and a curve fit was done on the data resulting in a third degree polynomial $\left(k_{\phi}\right)$ which is presented in the Appendix. The plot of the stiffness against the opening angle can be seen in

Table 6 Angles of the mechanism

\begin{tabular}{lllr}
\hline$\phi\left[^{\circ}\right]$ & $\beta\left[^{\circ}\right]$ & $\gamma\left[^{\circ}\right]$ & $\delta\left[^{\circ}\right]$ \\
\hline 0 & 18.612 & 70.374 & -22.247 \\
10 & 50.3072 & 49.8725 & -1.0533 \\
20 & 71.5579 & 33.1459 & 13.4708 \\
30 & 88.2593 & 17.1578 & 24.1841 \\
40 & 102.4986 & 0.9717 & 32.2373 \\
50 & 115.6444 & -16.1956 & 38.2158 \\
60 & 129.1891 & -35.7065 & 42.2497 \\
\hline
\end{tabular}




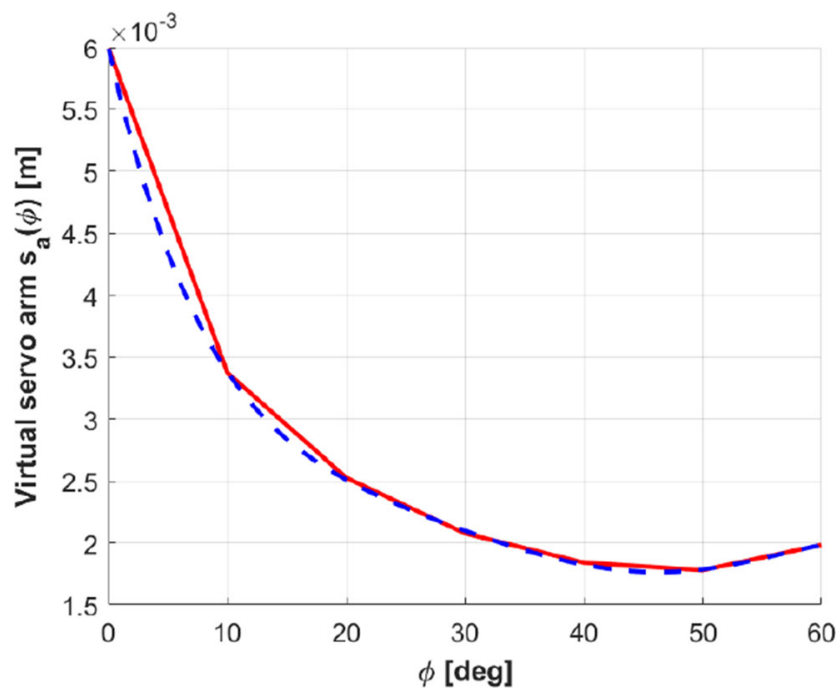

Fig. 38 Curve fit to airbrake angle virtual servo arm data (continuous line: original data, dashed line: fitted curve)

Fig. 42. The fitted formula can cover the whole angle range $0-60^{\circ}$ with reasonable non-negative values. As opening mechanism flexibility is present in the system its effects on the dynamic behavior should be examined. This is the topic of the next part.

\subsubsection{Effects of Airbrake Inertia and Mechanism Stiffness}

The inertial moment of the flap was estimated by modelling the flap as a rectangular prism with an external axis of rotation in distance $c=51.5 \mathrm{~mm}$, as illustrated in Fig. 43 . The length $l=218 \mathrm{~mm}$ is assumed corresponding to the edge length of the actual flap, the width $b$ according to

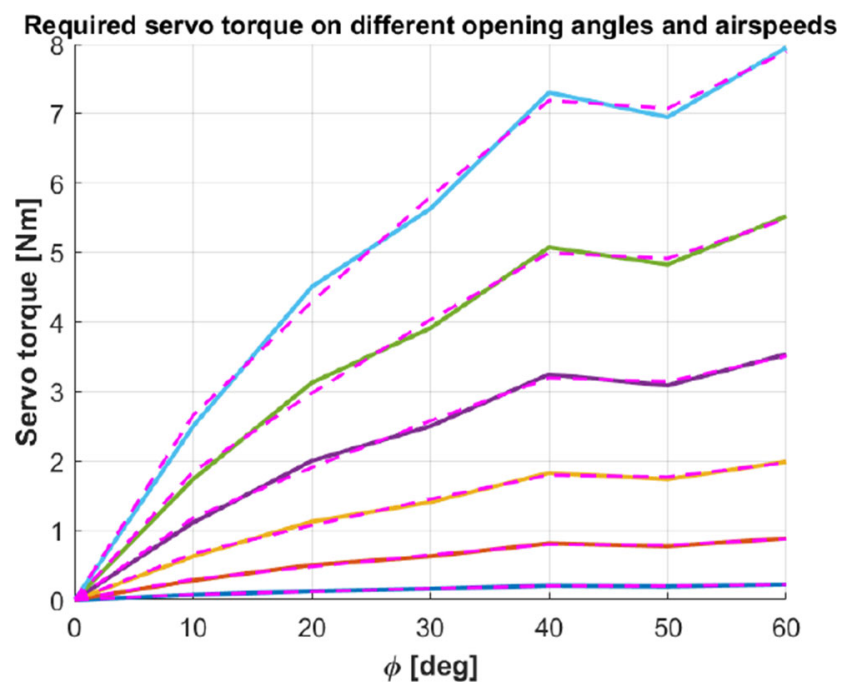

Fig. 39 Servo torque from mechanism (color continuous) and from approximation (magenta dashed). Lowest curve for $10 \mathrm{~m} / \mathrm{s}$ while highest for $60 \mathrm{~m} / \mathrm{s}$ airspeed

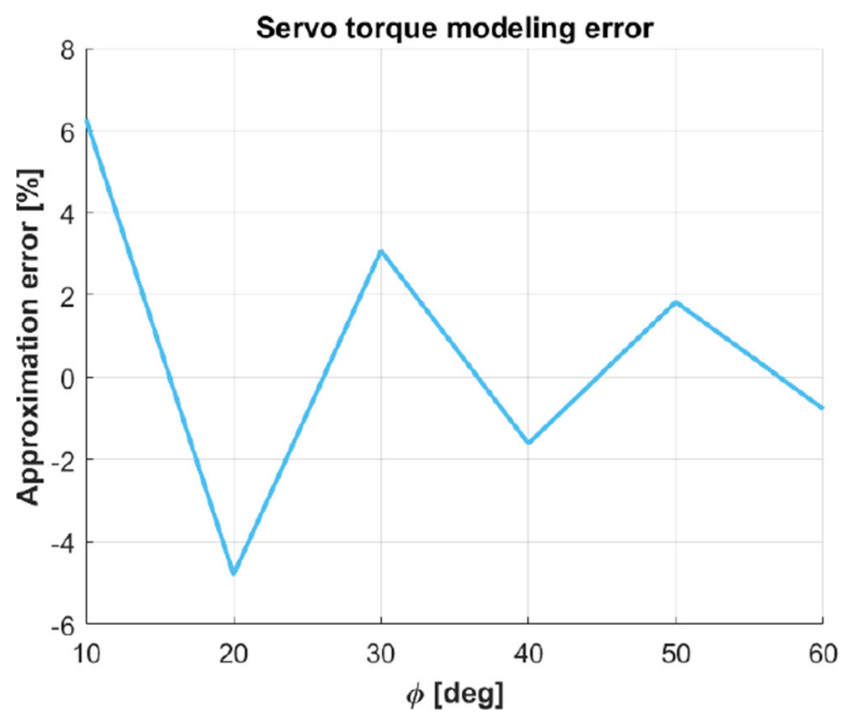

Fig. 40 Servo torque approximation errors

the mean width of the flap and the thickness $d=4 \mathrm{~mm}$ according to the actual sandwich core thickness of the structure. An isotropic density distribution is assumed. To compensate for the external axis of rotation, the parallelaxes theorem is used. The inertial moment can be estimated by using the following equation (see [7]):

$$
I=m\left(\frac{l^{2}}{12}+\frac{d^{2}}{12}\right)+m\left(\frac{l^{2}}{4}+c^{2}\right)
$$

With the second term being the compensation from parallel axes theorem and using the measured mass $m=$ $0.115 \mathrm{~kg}$ of the actual flap. As the formula shows the width (b) is not required to estimate the moment of inertia with regard to the given axis. Due to the slight trapezoidal planform of the flap, the actual center of gravity is closer to
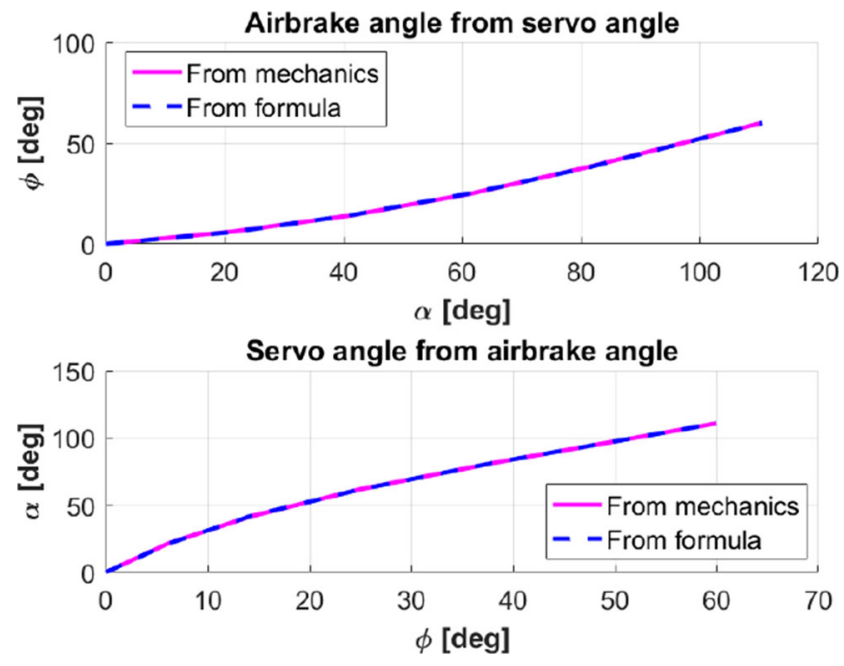

Fig. 41 Servo angle - airbrake angle curves 


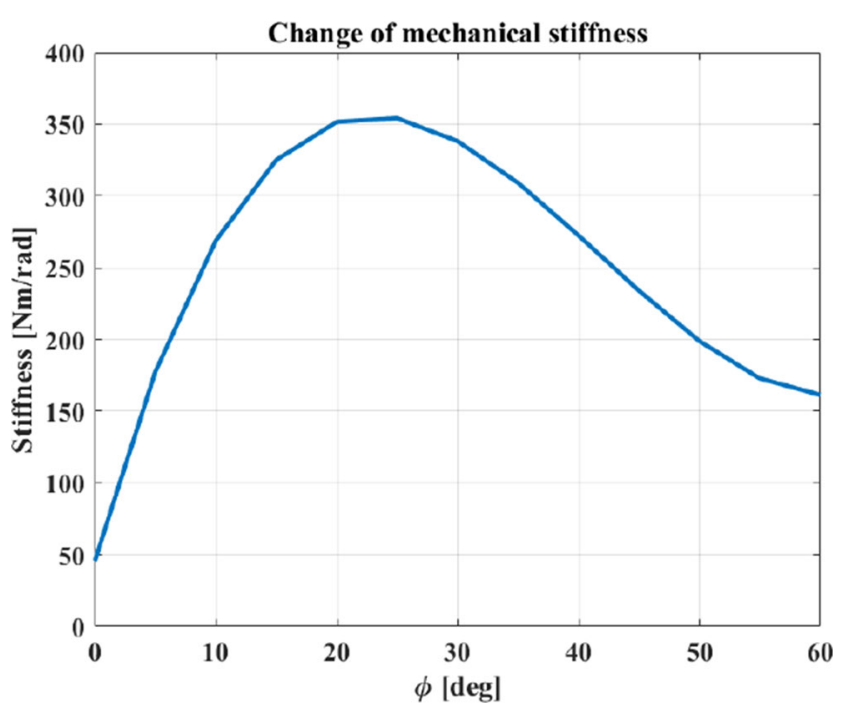

Fig. 42 Change of mechanical stiffness with opening angle

the axis of rotation. In consequence, the inertia is estimated a bit conservatively.

Figure 44 shows the simple dynamical model of the servo-airbrake setup considering the stiffness $k_{\phi}$ of the mechanism as a torsional spring. $\alpha$ is the servo arm angle ( $\beta$ ) shifted to be zero at minimum deflection as $\alpha=\beta-$ $18.612^{\circ}$ while $\phi$ is the deflection angle of the airbrake itself. For the relation between $\beta$ and $\phi$ through the airbrake deflection mechanism see Fig. 37. To simplify the model a one-to-one mapping between $\alpha$ and $\phi$ is assumed as any static gain between them would not modify dynamic behavior. The servo torque $T$ equals the elastic force in the mechanism at every time as: $T=k_{\phi}(\alpha-\phi)$.

The dynamic equation of the airbrake considering its mass moment of inertia $I=2.127 \cdot 10^{-3} \mathrm{kgm}^{2}$ the torque of the aerial forces on the airbrake $T_{L}$ and the fact that it is driven through the flexible mechanism results as:

$I \ddot{\phi}=k_{\phi}(\alpha-\phi)-T_{L}$

Note that here the airbrake load torque is transformed to the servo shaft $\left(T_{L}\right)$ and the stiffness of the mechanism is considered as a simple torsional spring between. Reorganizing the above expression and making Laplace transform of each side results in two transfer functions, one

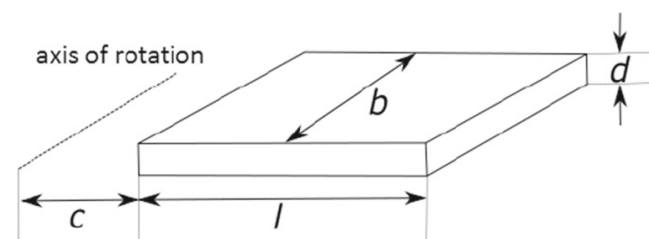

Fig. 43 Simple model of airbrake flap for inertia calculation

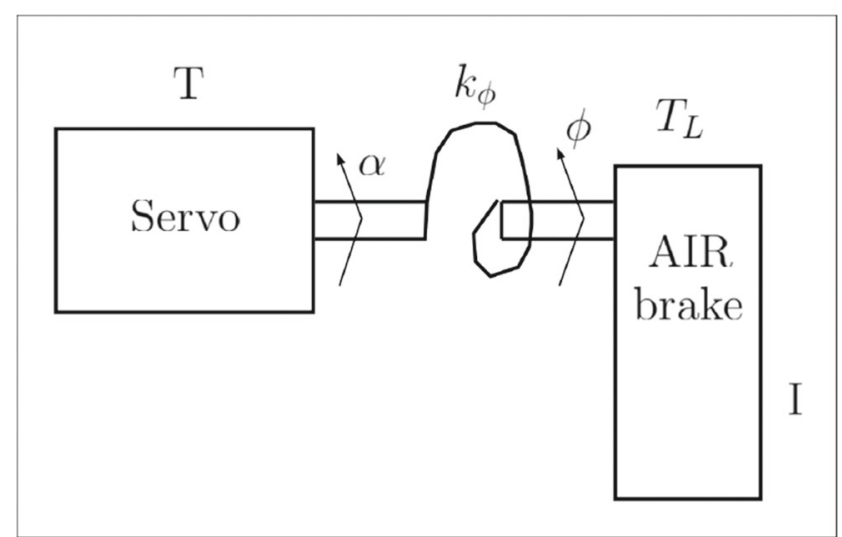

Fig. 44 Simple dynamic model with stiffness and inertia

from servo angle $\alpha$ to airbrake deflection $\phi: G_{\alpha}(s)$ and one from aerial load $T_{L}$ to airbrake deflection $\phi: G_{L}(s)$

$$
\begin{aligned}
I \ddot{\phi}+k_{\phi} \phi & =k_{\phi} \alpha-T_{L} \\
\phi(s) & =\frac{k_{\phi}}{I s^{2}+k_{\phi}} \alpha(s)+\frac{-1}{I s^{2}+k_{\phi}} T_{L}(s) \\
G_{\alpha}(s) & =\frac{k_{\phi}}{I s^{2}+k_{\phi}} \\
G_{L}(s) & =\frac{-1}{I s^{2}+k_{\phi}}
\end{aligned}
$$

So finally, one gets a two input ( $\alpha$ servo angle and $T_{L}$ torque of aerial forces) one output ( $\phi$ airbrake angle) system. Note that the $k_{\phi}$ term depends on the actual angle of the airbrake (see Fig. 42) and so these transfer functions should be examined for different airbrake angle values. Bode plots were plotted for angle values $0: 5^{\circ}: 60^{\circ}$ covering the whole range and shown in Figs. 45 and 46.

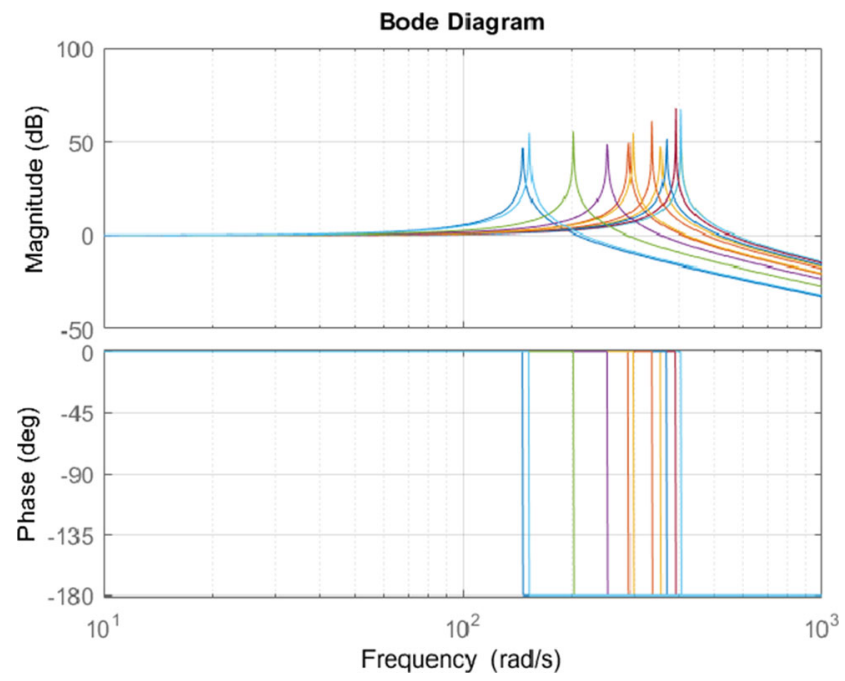

Fig. 45 Bode plots for servo angle $\alpha$ input 


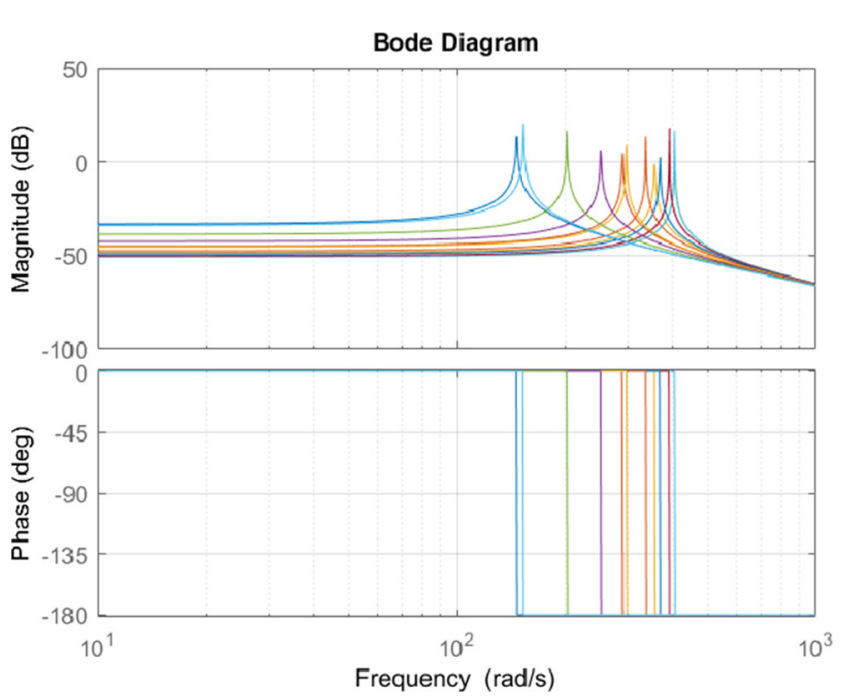

Fig. 46 Bode plots for airbrake load $T_{L}$ input

They show that both term can be well approximated with a constant gain on a wide frequency range. They give undamped oscillations only on high frequencies (well above $70 \mathrm{rad} / \mathrm{s}=11 \mathrm{~Hz}$ ) on which the airbrake will not be operated as its bandwidth is about $1.4 \mathrm{~Hz}$ shown by Fig. 11. Another fact to be considered is that the mechanism should also have damping which is unknown but surely present and so could damp the oscillations to a favorable level even on high frequencies. If flight testing does not show oscillations even with high frequency excitation then a static model including only the effect of stiffness can be used.

Considering low frequency approximations of the transfer functions one gets:

$\phi(s)=1 \cdot \alpha(s)+\frac{-1}{k_{\phi}} T_{L}(s)$
Taking the inverse Laplace transform and reorganizing the terms gives:

$T_{L}=k_{\phi}(\alpha-\phi)=k_{\phi} \Delta \phi$

Where $\Delta \phi$ is the angular deformation and can be obtained as $\Delta \phi=\alpha-\phi=\frac{T_{L}}{k_{\phi}}$. In the airbrake simulation the servo deflection $\alpha$ and the momentarily load $T_{L}$ on the servo are known and so $k_{\phi}$ and $\phi$ can be obtained as shown in the structure in Fig. 47.

\section{Full Airbrake Simulation Model and Test Results}

After identifying the model components the whole system with servo model, mechanism and aerodynamic effects was constructed and simulated in Matlab Simulink. The block scheme of the whole simulation structure is shown in Fig. 47. Here, Servo is the servo simulation model from Fig. 24, $p_{d y n}$ calculates the dynamic pressure from $\rho$ air density and $V$ airspeed, $\alpha(\phi)$ and $\phi(\alpha)$ are the polynomials transforming airbrake angle to servo angle and vice versa. $k_{\phi}$ is the angle dependent stiffness coefficient, the load torque $T_{L}$ is divided by it to get the angle change $(\Delta \phi)$ of the airbrake as derived at the end of Section 4.2. To be a bit more realistic the whole angle change is subtracted from the airbrake position used to calculate air drag and normal force, but only half of it is subtracted to calculate the virtual servo arm $s_{a}(\phi)$ assuming non-uniform deformation inside the mechanism. $S A T$ represents the saturation of the airbrake angle output between $0 \mathrm{rad}$ and 1.0472 rad (0$60^{\circ}$ ). The blocks with three $\times$ represent the multiplication of all inputs. $D$ is the air drag force as the useful output of the system contributing to the deceleration of the aircraft.

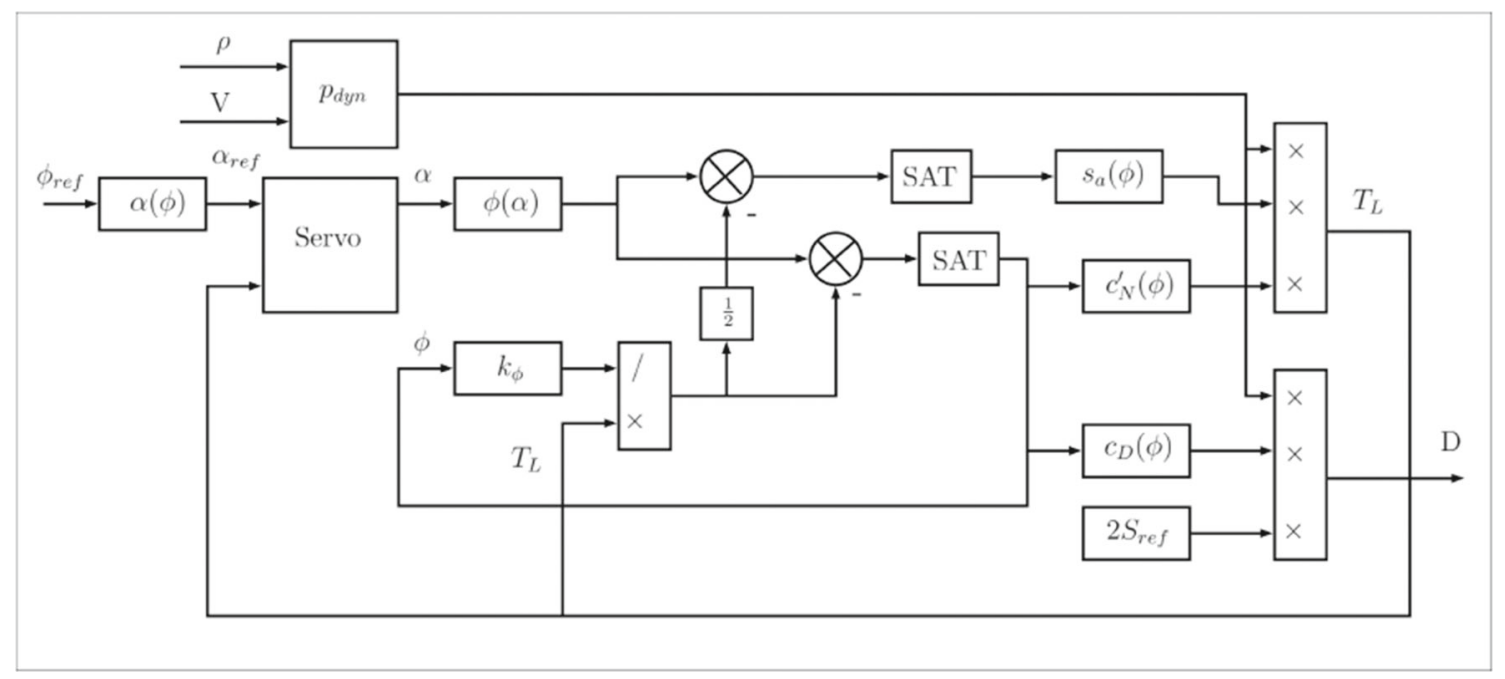

Fig. 47 Simulink model of the whole airbrake 
After the construction the model was tested with step airbrake angle reference changes, airspeed and air density (flight altitude) sweeps and even with chirp signal input. The overall system was stable and realistic in all cases so it was built into the nonlinear simulation model of the FLEXOP aircraft. These test cases are not presented in detail here to hold the length of the paper acceptable, rather the final SIL simulation results of the full aircraft nonlinear model are presented.

\subsection{Airbrake Model Verification in SIL Mission Simulation}

The full airbrake model was integrated into the nonlinear simulation model of the FLEXOP aircraft by DLR (Deutsches Zentrum für Luft- und Raumfahrt e.V.) and then the baseline controller of the aircraft [14] was completed with a simple logic which fully opens and then closes the airbrake to help deceleration as the aircraft approaches the end of the test leg. The baseline controller is a cascade gain-scheduled PID control with several inner and outer loops which is detailed in [14]. Giving more details is not the topic of this article. It is possible that in the future the airbrake operation logic will be replaced by a more sophisticated controller but if flight test results with the logic will be satisfactory then this won't be required. At first, the standalone airbrake model deflection and load values were compared to the SIL results to check if the integration of the model into the nonlinear simulation is flawless. The standalone model is driven by deflection commands and dynamic pressure saved from the SIL simulation. Airbrake load, servo and airbrake deflections are compared in Figs. 48 and 49. In Fig. 48 'SIL' means the result from the nonlinear SIL simulation of the FLEXOP aircraft while 'Standalone' means other results from the standalone simulation. 'servo' means the deflection of the servo arm while 'airbrake' means deflection of the airbrake. The load curves are almost exactly the same while the deflections show different transient responses but otherwise they perfectly match. The SIL airbrake and servo deflections have some over- and undershoot in the transient while the standalone ones do not. This is caused by the different solver settings in the standalone (fixed step, discrete) and SIL (fixed step, ode3) Matlab Simulink simulations as the ode3 solver is iterative. In conclusion the airbrake model integration is flawless the nonlinear model can be used for controller tuning, SIL and hardware-in-the-loop (HIL) tests.

The next step after the verification of airbrake integration into the model was to test its performance accelerating the demonstrator to different flight speeds and then decelerating it with or without airbrake. The nominal cruise speed of the demonstrator is $38 \mathrm{~m} / \mathrm{s}$ and the airbrake is started to be closed as the speed decreases to $39 \mathrm{~m} / \mathrm{s}$ during

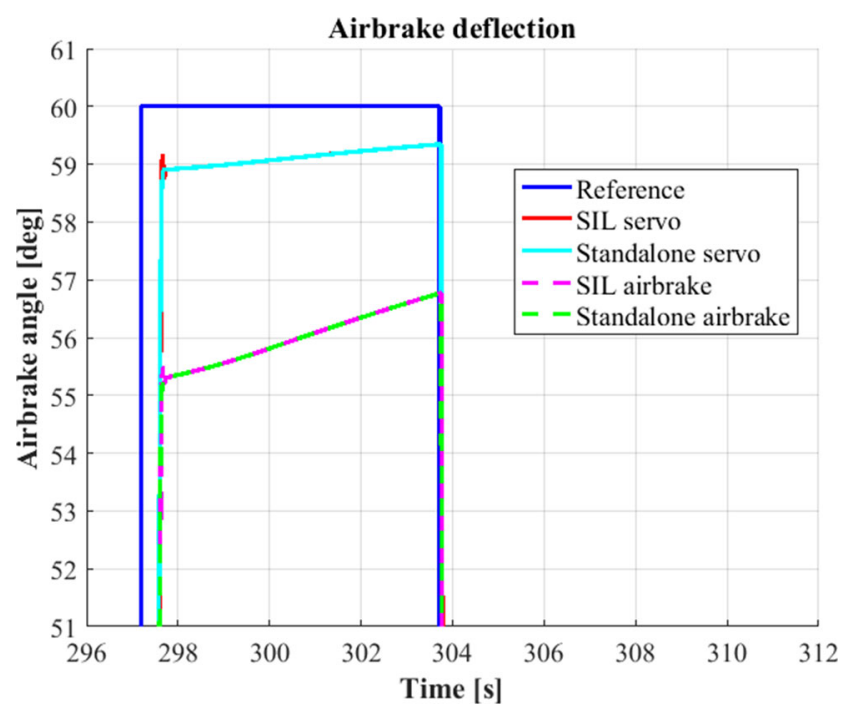

Fig. 48 Airbrake deflection angles from standalone simulation and SIL

the deceleration. SIL tests were done guiding the aircraft along the racetrack test pattern at 300m AGL applying accelerations to 42,46 and $50 \mathrm{~m} / \mathrm{s}$ at each test leg representing the gradual increase of flight speed during the evaluation of the flutter controller (note that the flutter onset speed is about $51 \mathrm{~m} / \mathrm{s}$ ).

The airspeed characteristics are plotted in Figs. 50, 51 and 52. The without airbrake airspeed curves are shifted to start deceleration at the same time as the curves with airbrake application. That's why they seem to react to the initial reference change too late. The figures show that with the application of the airbrake the airspeed can reach the 38 $\mathrm{m} / \mathrm{s}$ cruise value earlier with smaller or no overshoot.

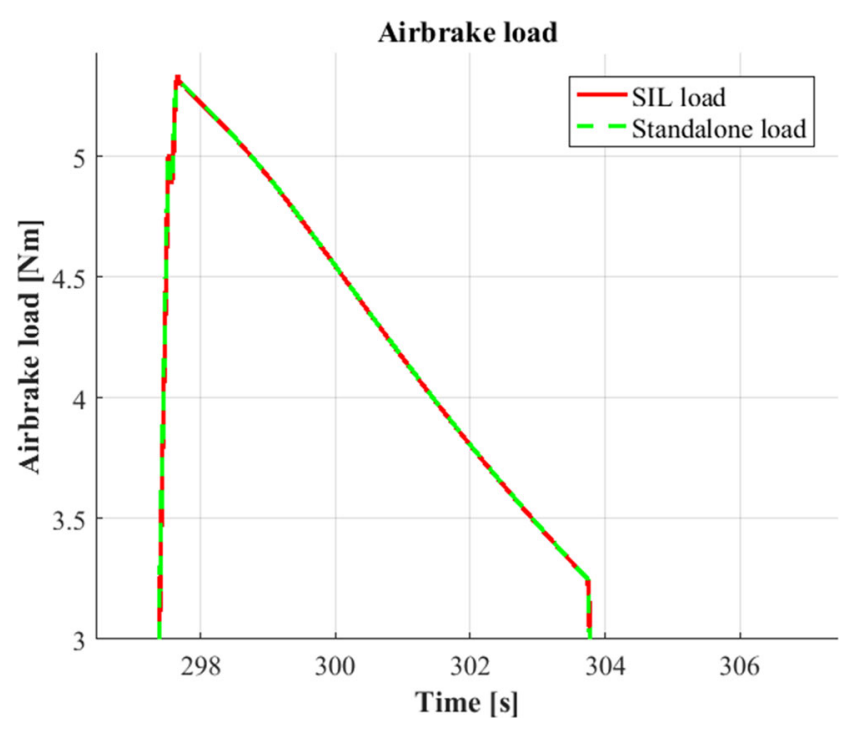

Fig. 49 Airbrake load from standalone simulation and SIL 


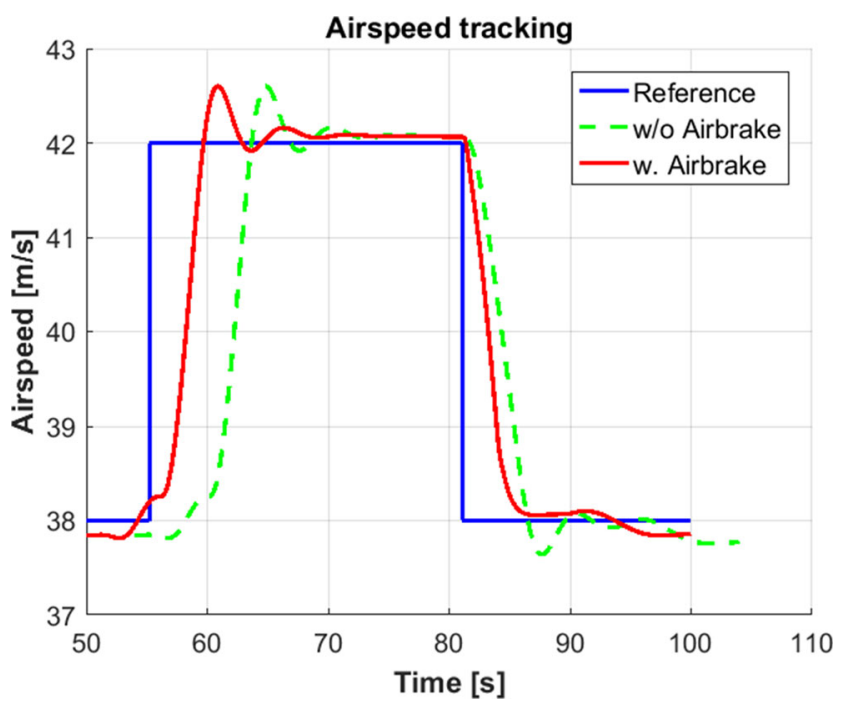

Fig. 50 Airspeed profiles with $42 \mathrm{~m} / \mathrm{s}$ reference

Table 7 summarizes the settling times of SIL simulation regarding $\pm 2 \%$ tolerance around the $38 \mathrm{~m} / \mathrm{s}$ cruise speed $(37.24-38.76 \mathrm{~m} / \mathrm{s}$, the usually applied $\pm 5 \%$ range was too large with almost $40 \mathrm{~m} / \mathrm{s}$ upper bound). The time gain means the time with which the deceleration with airbrake is shorter and so the flutter test can be longer. The distance gain is the time gain multiplied by $38 \mathrm{~m} / \mathrm{s}$ which is the minimum gained distance on the test leg. The table shows that the flutter test can be from $1.5 \mathrm{~s}$ to as large as almost $4 \mathrm{~s}$ longer with airbrake application which can largely help to better evaluate the behavior. After SIL testing the final verification of the airbrake model is done in the next section considering flight test results.

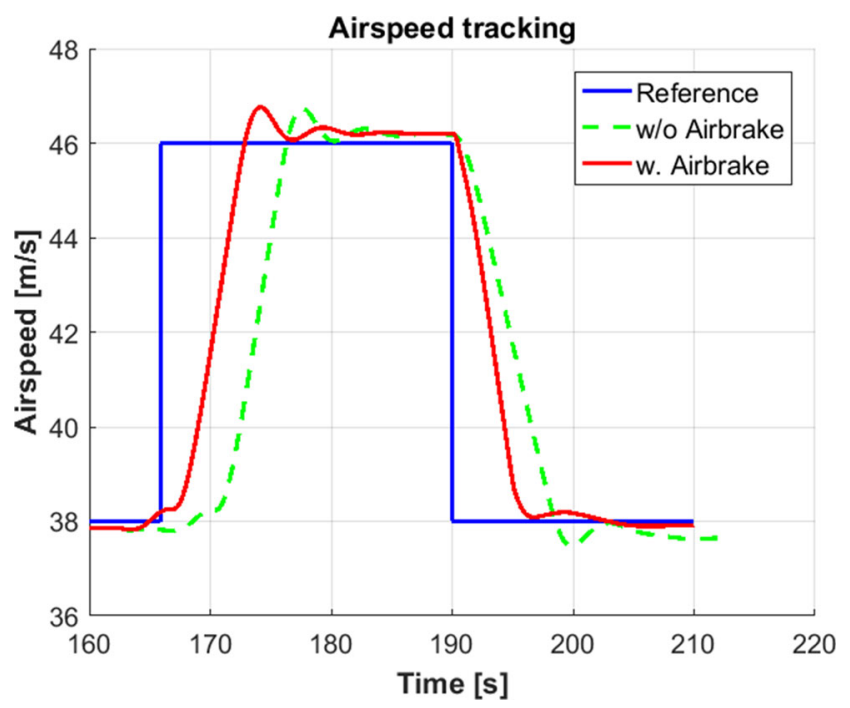

Fig. 51 Airspeed profiles with $46 \mathrm{~m} / \mathrm{s}$ reference

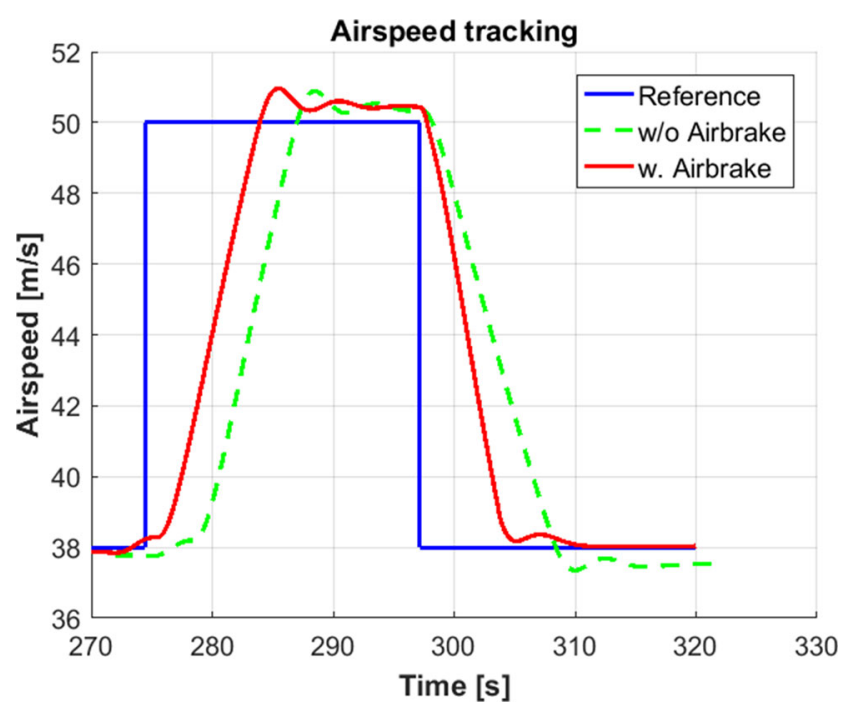

Fig. 52 Airspeed profiles with $50 \mathrm{~m} / \mathrm{s}$ reference

\subsection{Flight Test Results}

Real flight test results were obtained in two manual flights in August 2019 at München Oberpfaffenhofen airport. The aircraft was manually controlled into almost straight and level flight and then applying constant throttle (see Fig. 60) and trying to hold the flight direction (see Fig. 53) the airbrake was manually fully opened and closed giving a staircase reference until maximum deflection (see e. g. Fig. 54) and after some time back. Straight and level flight was targeted to give an opportunity to realistically evaluate the effect of the airbrake in decreasing the airspeed of the aircraft. The tests are approximately repeatable as the similar altitude and airspeed ranges show in Table 8 . Unfortunately manual control can not perfectly hold altitude (see Fig. 59) so finally the system specific energy was used for evaluation. In the future in frame of the next FLiPASED (see [1]) project airbrake tests are planned with autopilot control during 2020 which will better be repeatable. However, precise evaluation of airbrake effectiveness is not strictly required to evaluate the airbrake dynamic model itself that's why model identification results can be published now.

The ranges of barometric flight altitude $(h)$ and indicated airspeed $(V)$ are shown in Table 8 and Fig. 59 shows their relative changes starting from the first value at activation

Table 7 Airspeed settling absolute times in SIL

\begin{tabular}{llll}
\hline$V_{\text {ref }}[\mathrm{m} / \mathrm{s}$ ] & 42 & 46 & 50 \\
w/o Aibrake [s] & 85.6 & 197.73 & 307.64 \\
w. Airbrake [s] & 84.1 & 195.06 & 303.85 \\
Time gain [s] & 1.5 & 2.67 & 3.79 \\
Distance gain [m] & 57 & 101.46 & 144.02 \\
\hline
\end{tabular}




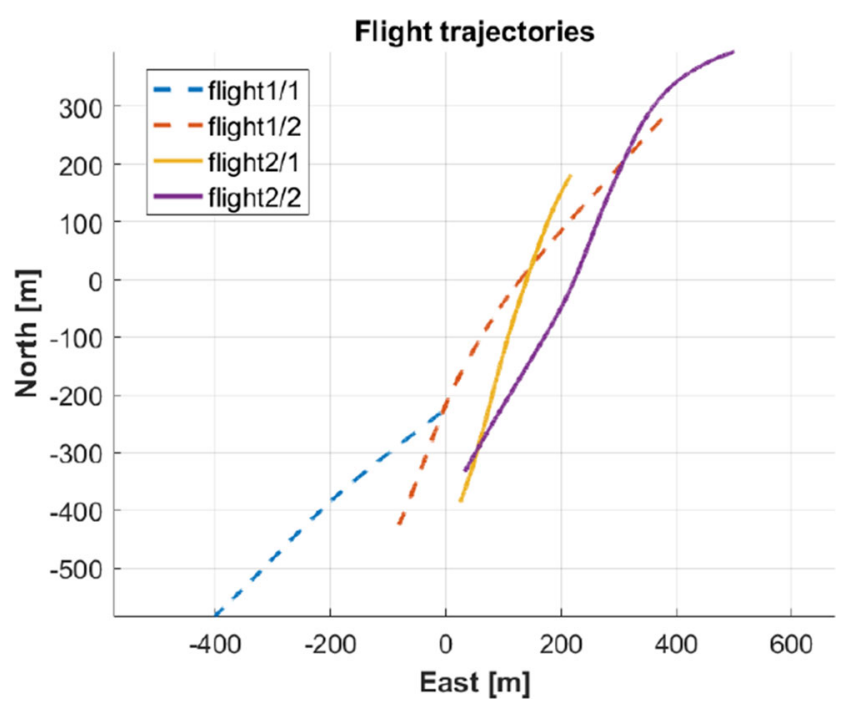

Fig. 53 Flight trajectories during airbrake tests

of airbrake. The figure well shows that these ranges do not cover monotonically increasing or decreasing values, both parameters can decrease and increase even multiple times during one airbrake application test section. That's why later airbrake effectiveness is evaluated based-on the specific energy of the system because there is no definite change of the airspeed and/or altitude. Figure 53 shows the flight trajectories which are close to straight during airbrake application. In autopilot controlled flight the direction hold of the aircraft will be much better than here in manual flight.

The airbrake was fully opened and then closed at constant throttle value (see Fig. 60). As the on-board system saves the RC (radio controller) commands and measures the deflections of the actuators its possible to plot the command and answer together and simulate the airbrake deflection

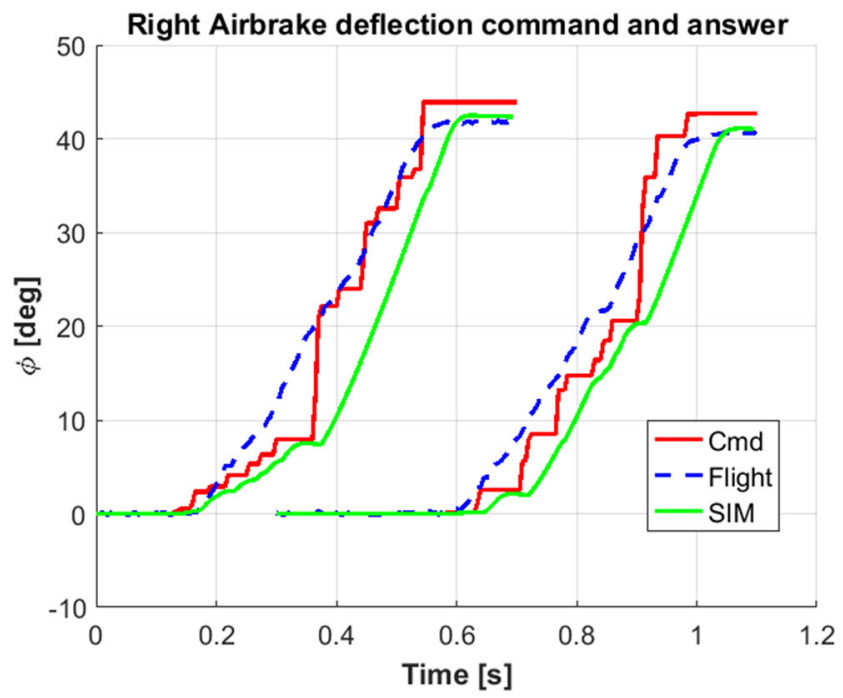

Fig. 54 Opening of right airbrake in 2nd flight ( $\mathrm{Cmd}=$ manual command, Flight $=$ in-flight measurement, $\mathrm{SIM}=$ simulation result)
Table 8 Airbrake flight test conditions

\begin{tabular}{lllll}
\hline Parameter & flight 1/1 & flight 1/2 & flight 2/1 & flight 2/2 \\
\hline $\mathrm{h} \mathrm{[m]}$ & $633-655$ & $597-618$ & $586-600$ & $668-692$ \\
$\mathrm{~V}[\mathrm{~m} / \mathrm{s}]$ & $32.8-36.2$ & $32-36.7$ & $32-43$ & $33-44$ \\
\hline
\end{tabular}

based-on the command and the also logged barometric altitude and airpseed. Basically the servo deflections are commanded and measured but the airbrake opening angle is considered in the calibration characteristics. Examining the in-flight opening characteristic (see Fig. 54) shows that the opening position seems to occur before the command which is physically impossible. Taking a closer look shows that the start and end of opening is after the command starts and ends so the overall too early opening should be virtual only because of the large quantization error of saved command. This is underlined by the closing characteristic (see Fig. 55) where the airbrake deflection is after the command. Note that the manual command is highly quantized while the airbrake angle output is a smooth signal so the command on the servo should be smoother than the logged signal. This means that the airbrake simulation results will be quantized which should be neglected in the model comparison. Also in the opening cases the lag of the simulated curve is realistic relative to the command while seems to be too late relative to the in-flight deflection.

Figure 54 also shows that the rise time is about $0.3-0.35 s$ which is a bit higher than the $\approx 0.25 s$ value from Table 2 related to the about $3 \mathrm{Nm}$ load resulting (consider Fig. 39) from the about $40 \mathrm{~m} / \mathrm{s}$ airspeed in flight 2 during airbrake opening.

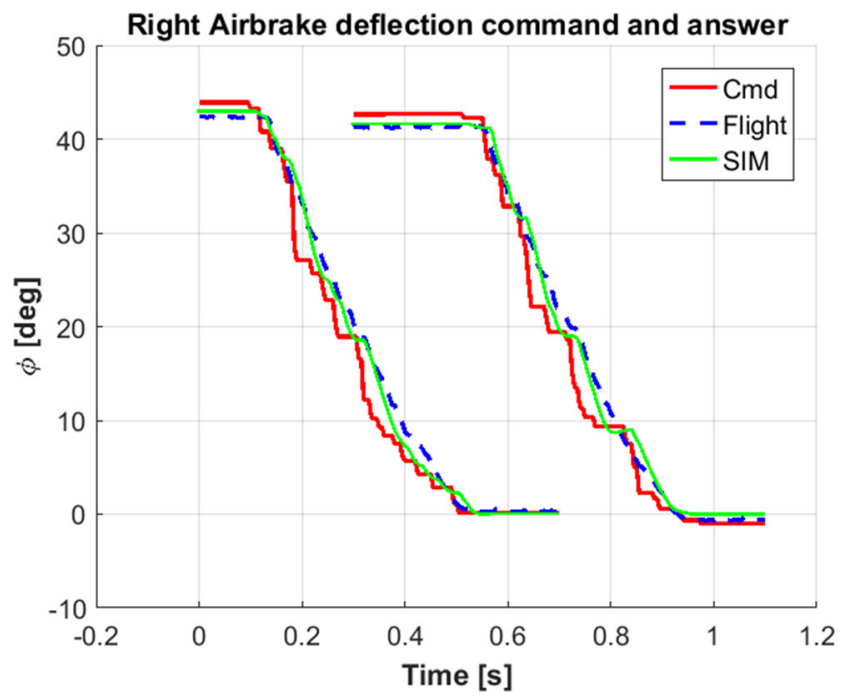

Fig. 55 Closing of right airbrake in 2nd flight ( $\mathrm{Cmd}=$ manual command, Flight $=$ in-flight measurement, SIM = simulation result) 
The airbrake model was simulated considering the actual barometric flight altitude and indicated airspeed values and the manual deflection commands of the airbrake. Note that the maximum physical deflection of the airbrakes on the aircraft is about $45^{\circ}$ in contrast to the maximum $60^{\circ}$ test bench deflection. The steady state values of simulated and in-flight airbrake angles are very close to each other (max $0.6^{\circ}$ difference) so this does not require model refinement.

The comparison of simulated closing characteristic (as the opening can not be taken as a precise reference) with the in-flight one shows that the simulation with the three time steps delay in the servo dynamics lags more than the flight data. This means that the delay could be decreased finally to 1 step giving results better following the flight data (see Fig. 56). The figure also shows that the slope of the simulated signal is a bit larger than the in-flight one. This can be possibly solved by further limiting the maximum closing speed of the servo under load. Examining the closing speed limits and the load resulting from inflight dynamic pressure shows that the load is about $2 \mathrm{Nm}$ and so the current maximum closing speed is $-372^{\circ} / \mathrm{s}$ in the simulation model. The maximum limitation of the speed can be $-340^{\circ} / \mathrm{s}$ but this is very close to the current model $(91.3 \%)$ and does not improve significantly the performance so finally the closing speed characteristic remained unchanged. As the airbrake closes only if the airspeed is decreased to a low value the load during the closing will always be small so the load dependent closing speed will be around the above mentioned values.

Considering the slope of the opening in Fig. 54 in the first case the simulated slope is a bit larger while in the second

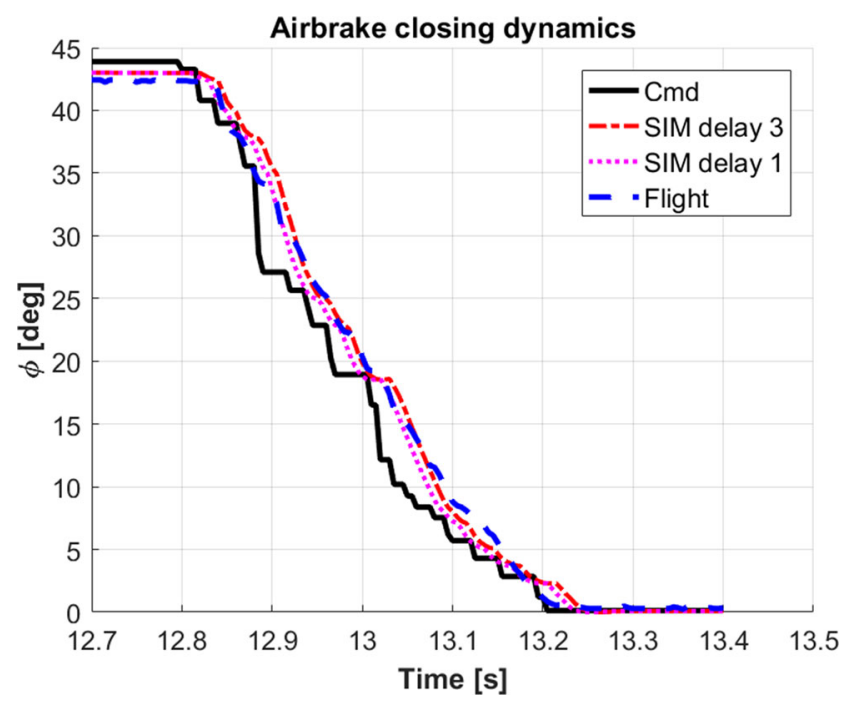

Fig. 56 Simulation with different delays compared to flight results $(\mathrm{Cmd}=$ manual command, Flight $=$ in-flight measurement, $\mathrm{SIM}=$ simulation result)
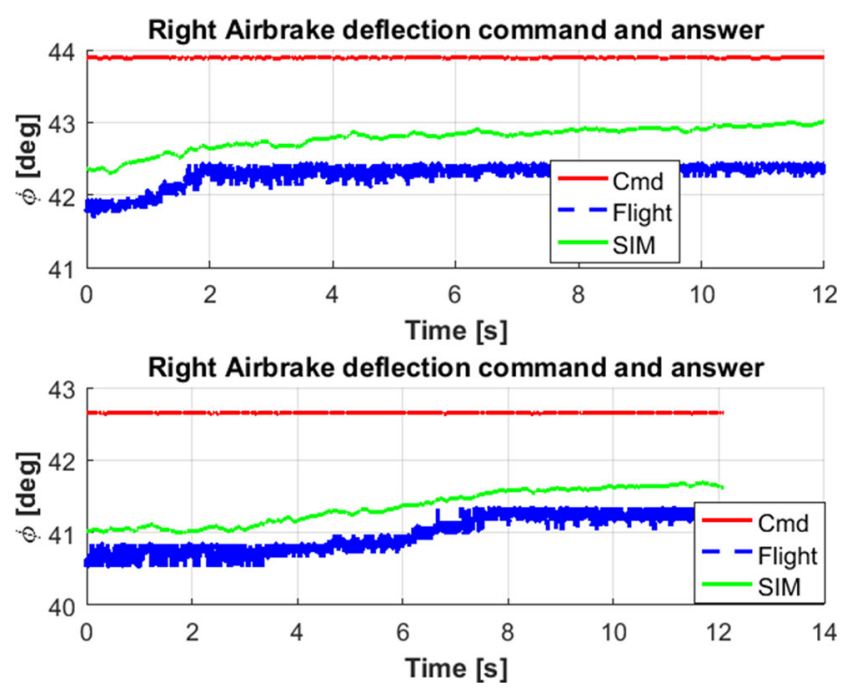

Fig. 57 Change of airbrake position while the reference is constant $(\mathrm{Cmd}=$ manual command, Flight $=$ in-flight measurement, $\mathrm{SIM}=$ simulation result)

case its almost the same as the in-flight one so finally the opening angular velocity limits are unchanged.

Figure 57 shows that both the in-flight and the simulated data has a change in airbrake position when the manual deflection command is constant. This should be caused by the decrease in airbrake load and is well followed by the simulation model. This verifies the approach to identify also the load to servo angle transfer function $G_{T_{L}}(z)$ and excludes the possibility of having this effect only due to test bench measurement errors.

Figure 58 shows the commanded and real deflections of the left airbrake in the 2nd flight. Similar half doublet deflections were applied in all test cases and the airspeed, altitude and specific energy results are obtained with these

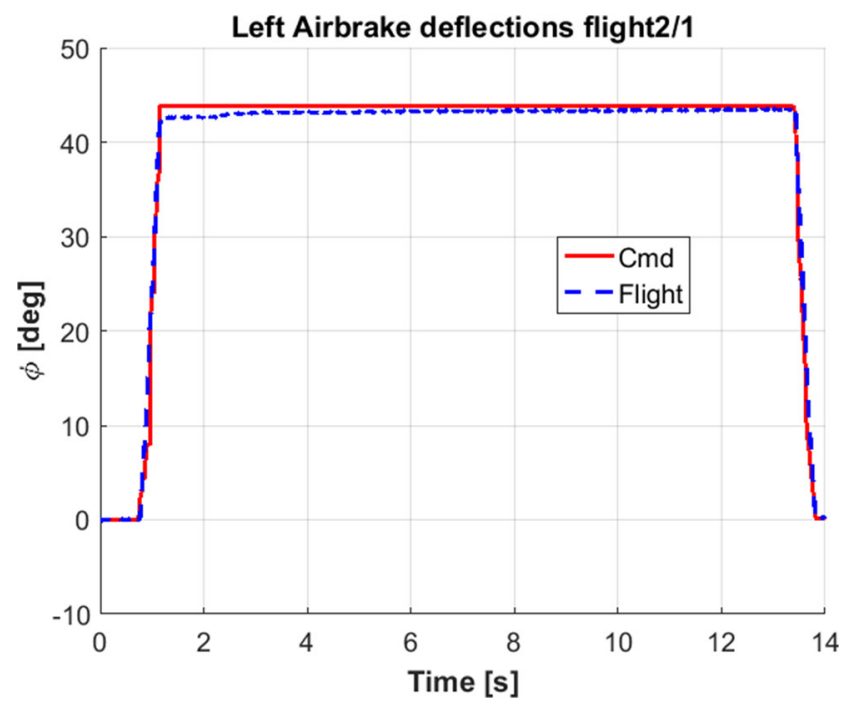

Fig. 58 Left airbrake deflection in 2nd flight 
14 to 24 seconds deflections. Figure 59 shows the change in airspeed and altitude during airbrake application. All initial values were shifted to zero to show the decrease or increase of the values relative to the initial. The airspeed decreases with about $2 \mathrm{~m} / \mathrm{s}$ in the first flight and more then $5 \mathrm{~m} / \mathrm{s}$ in the second while the time horizons are about the same. This is because in the first flight the initial airspeeds are $34-36 \mathrm{~m} / \mathrm{s}$ while in the second flight they are about $43 \mathrm{~m} / \mathrm{s}$ (see Table 8). As the drag force of the airbrake scales quadratically with airspeed this is not surprising as higher drag means higher deceleration. Regarding the altitude it increases in one of the cases while decreases in all other three. As the altitude can increase / decrease during the maneuvers its hard to estimate if the decrease in airspeed is caused by altitude change or by the airbrake.

To decide about this the overall energy state of the aircraft should be considered. With constant throttle and small changes in altitude and airspeed the thrust of the jet engine can be assumed to be almost constant and so starting from a trimmed condition the energy content should not change significantly. However, plotting the specific energy $u=g h+\frac{1}{2} V^{2}$ (see Fig. 60, $h$ is barometric altitude and $g$ is the gravitational constant) shows that the energy content of the aircraft decreases so the airbrake removes energy from the system. As the mass of the aircraft slightly changes due to fuel consumption the specific energy is considered instead of the whole potential and kinetic energies.

As a conclusion it can be stated that the flight test data verifies the airbrake simulation model having similar results in simulation then in flight so this model can be applied for more sophisticated airbrake control design and for the HIL verification of any autopilot applying airbrake.
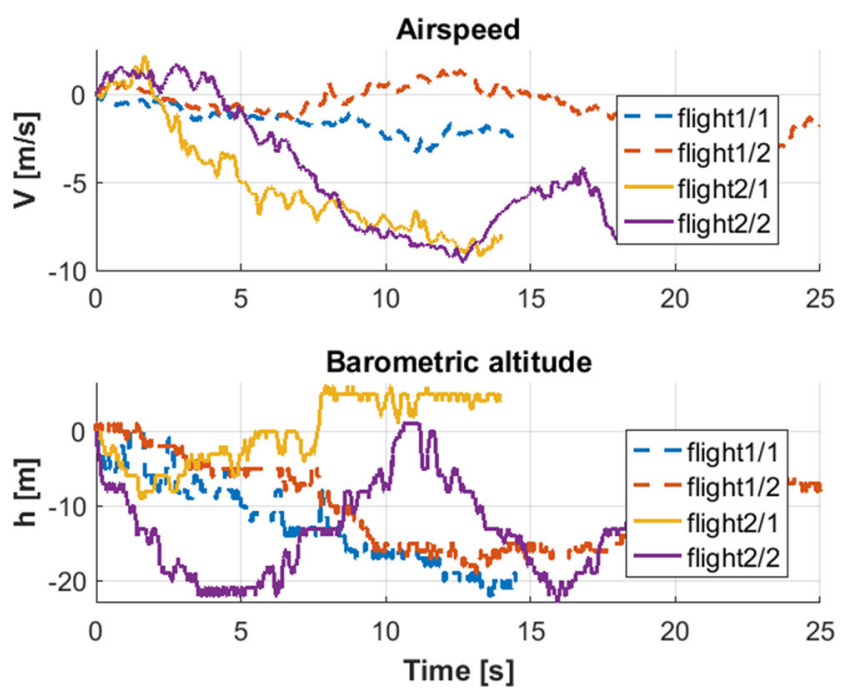

Fig. 59 Airspeed and barometric altitude during airbrake operation

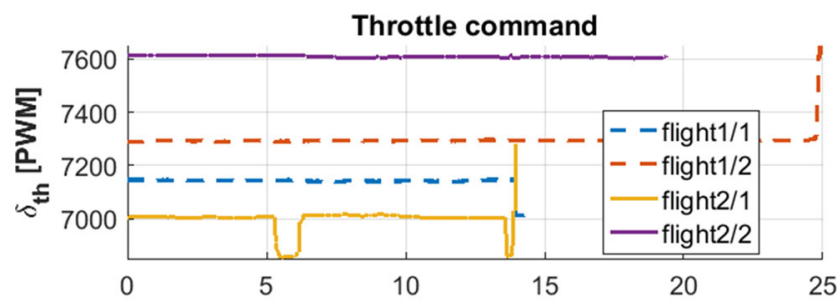

Actual specific energy level

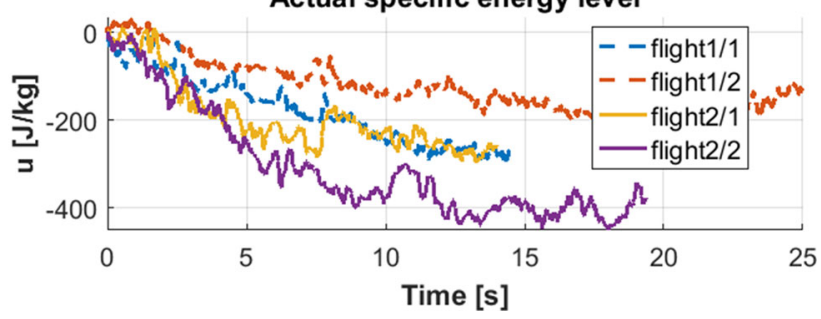

Fig. 60 Throttle positions $\delta_{t h}$ and specific energy $u$

\section{Conclusion}

This paper presents the modeling and system identification of the airbrake of an unmanned experimental aircraft. This airbrake consists of a servo motor, a nonlinear mechanism and the aibrake flap itself. After briefly introducing the demonstrator aircraft and summarizing the design considerations the system identification concept is discussed. Then the applied test benches are briefly described which are a full scale mock-up and a servo test bench with load motor.

From this point the work focuses on the identification of the servo dynamics based on test bench measurements first pulling out the significant nonlinearities from the system. These include the characterization of the load dependent opening and closing angular velocities and the estimation of the saturation level of servo angular velocity reference input. The remaining linear part of the servo dynamics was identified as a transfer function plus delay term. As the load causes a steady-state servo angle change this load dependent angle change was also modeled as a transfer function. This effect was verified by flight test results so this transfer function is a required part of the airbrake model. After constructing the servo model its verification based-on test bench measurements was done. The next step was the description and identification of static characteristics. This includes the aerodynamics as drag and normal force coefficients. The former is modeled with a line fit against the airbrake deflection angle, the latter is normalized and modeled as a combination of a parabola and a line again against the airbrake angle. Then a virtual servo arm is defined and its characteristics are determined considering the nonlinearities in the airbrake opening mechanism. This, together with the normalized normal force coefficient gives the relation between the dynamic pressure, airbrake opening angle and servo load 
torque. After generating these the airbrake opening angle - airspeed - servo load torque characteristics are plotted for the whole operational range of the aircraft. The overall precision of this modeling resulted between 6 to 1 percent depending on the opening angle (compared to the calculated tabular data). These characteristics made it possible to select the required measurements for system identification which represent operational cases from a set of test bench measurements (opening angle range and load range sweep). Finally, the servo angle to airbrake angle and the reverse conversion characteristics were calculated by fitting polynomials and the angle dependent stiffness of the opening mechanism was also characterized. Before construction of the whole airbrake model the effect of opening mechanism stiffness and airbrake inertia on the dynamics was examined. It has turned out that this can cause oscillations only on very high frequencies which are outside the planned operating range and estimated bandwidth of the airbrake.

The whole airbrake simulation model was first checked in Monte-Carlo simulation with several angle references, airspeeds and flight altitudes and showed realistic results. Then, the airbrake model was integrated into the nonlinear simulation model of the FLEXOP demonstrator aircraft and its effect on the deceleration characteristics was examined considering software-in-the-loop trajectory tracking with a baseline controller. It has turned out that airbrake application can gain 1.5 to 4 seconds additional time to test the flutter controller of the aircraft.

Finally, in-flight manual test of the airbrake was done in August 2019. Comparing the simulation model with the inflight results has shown that the delay of the servo model can be decreased (from 3 time steps to 1) but otherwise the model well covers the opening, the closing and even the load change characteristics of the real airbrake. As in manual flight there is no precise altitude hold but the throttle and so the thrust was approximately constant the specific energy of the aircraft was examined during the application of the airbrake. This shows that the airbrake decreased overall system energy in all of the cases so its application in deceleration is advantageous.

Acknowledgements The authors gratefully acknowledge the contribution of Matthias Wuestenhagen at Deutsches Zentrum für Luft- und Raumfahrt e.V. (DLR) Institut für Systemdynamik und Regelungstechnik (RMC-SR-FLS) who integrated the airbrake simulation model into the nonlinear model of the FLEXOP aircraft.

The authors gratefully acknowledge the help of Tamas Luspay (senior research fellow, SZTAKI) in simulating the airbrake dynamics together with the baseline controller.

The authors gratefully acknowledge the contribution of Institute of Aircraft Design, Department of Mechanical Engineering, Technical University of Munich flight test team (Christian Roessler, Fabian Wiedemann, Sebastian Koeberle, Julius Bartasevicius and Daniel Teubl) with executing the flight tests.

Funding Information Open Access funding provided by ELKH Institute for Computer Science and Control.

\section{Compliance with Ethical Standards}

Conflict of interests The authors declare that they have no conflict of interest.

Open Access This article is licensed under a Creative Commons Attribution 4.0 International License, which permits use, sharing, adaptation, distribution and reproduction in any medium or format, as long as you give appropriate credit to the original author(s) and the source, provide a link to the Creative Commons licence, and indicate if changes were made. The images or other third party material in this article are included in the article's Creative Commons licence, unless indicated otherwise in a credit line to the material. If material is not included in the article's Creative Commons licence and your intended use is not permitted by statutory regulation or exceeds the permitted use, you will need to obtain permission directly from the copyright holder. To view a copy of this licence, visit http://creativecommons. org/licenses/by/4.0/.

\section{Appendix}

$$
\begin{aligned}
& \phi-c_{D} \text { fit: } \\
& c_{D}(\phi)=0.88 \cdot \phi[\mathrm{rad}] \\
& \qquad-c_{N}^{\prime} \text { fit: } \\
& c_{N_{L}}^{\prime}(\phi)=\phi^{2}\left[\mathrm{rad}^{2}\right]+1.8706 \phi[\mathrm{rad}] \text { if } 0 \leq \phi<0.6962 \mathrm{rad} \\
& c_{N_{H}}^{\prime}(\phi)=0.0514 \phi[\mathrm{rad}]+1.7512 \text { if } 0.6962 \mathrm{rad} \leq \phi \quad(7) \\
& \begin{aligned}
& \phi-s_{a} \text { fit: } \\
& s_{a}(\phi)[\mathrm{m}]=-0.02735 \phi^{5}\left[\mathrm{rad}^{5}\right]+0.09069 \phi^{4}\left[\mathrm{rad}^{4}\right]-0.11428 \phi^{3}\left[\mathrm{rad}^{3}\right] \\
&+0.071245 \phi^{2}\left[\mathrm{rad}^{2}\right]-0.02437 \phi\left[\mathrm{rad}^{2}\right]+0.006
\end{aligned}
\end{aligned}
$$

$\alpha-\phi$ curve:

$\phi(\alpha)[\mathrm{rad}]=-0.01864 \alpha^{3}\left[\mathrm{rad}^{3}\right]+0.213425 \alpha^{2}\left[\mathrm{rad}^{2}\right]+0.20056 \alpha[\mathrm{rad}]$

$\phi-\alpha$ curve:

$$
\begin{aligned}
\alpha(\phi)[\mathrm{rad}]= & -1.83448 \phi^{4}\left[\mathrm{rad}^{4}\right]+5.03271 \phi^{3}\left[\mathrm{rad}^{3}\right] \\
& -5.28037 \phi^{2}\left[\mathrm{rad}^{2}\right]+3.9602 \phi[\mathrm{rad}]
\end{aligned}
$$

$$
\text { (1) }
$$


System dynamics from delayed load to angle:

$G_{T_{L}}(z)=\frac{-0.002181}{1-0.5267 z^{-1}}$

\section{References}

1. Flight phase adaptive aero-servo-elastic aircraft design methods. cordis.europa.eu/project/id/815058

2. Flutter Free Flight Envelope Expansion for Economical Performance Improvement (2015). https://flexop.eu/

3. Adam, E., Guestrin, E.: Identification and robust control of an experimental servo motor. ISA Transactions 41(2), 225-234 (2002). https://doi.org/10.1016/S0019-0578(07)60082-2. http:// www.sciencedirect.com/science/article/pii/S0019057807600822

4. Anastasopoulos, L., Hornung, M.: Design of a real-time test bench for UAV servo actuators. AIAA AVIATION Forum. American Institute of Aeronautics and Astronautics. https://doi.org/10.2514/ 6.2018-3735 (2018)

5. Batista, J., Sousa, K., Nunes, J.L., Sousa, R.S., Thé, G.A.P.: Identification of the parameters of a ac servomotor using genetic algorithm. https://doi.org/10.5281/zenodo.1100140 (2015)

6. Davies, H., Kirk, F.: A resume of aerodynamic data on airbrakes. Tech. Rep., Aeronautical Research Council, Ministry of Supply (1951)

7. Grote, K.H., Feldhusen, J.: Dubbel: Taschenbuch Für Den Maschinenbau, Auflage, vol. 22. Springer, Berlin (2007)

8. Ishak, N., Abdullah, N.I., Rahiman, M.H.F., Samad, A.M., Adnan, R.: Model identification and controller design for servomotor. In: 2010 6th International Colloquium on Signal Processing and its Applications, pp. 1-4 (2010). https://doi.org/10.1109/CSPA.2010. 5545294

9. Koerner, D.: Experimental System Identification of an Electric Actuated Airbrake System for the FLEXOP Research UAV. Bachelor Thesis, Institute of Aircraft Design Technical University of Munich (2018)

10. KST: X30-12-1500 Technical Specification (2018)

11. Ljung, L.: System Identification: Theory for the User. Prentice Hall information and system sciences series. Prentice Hall PTR. https://books.google.hu/books?id=nHFoQgAACAAJ (1999)

12. Ortiz, X., Rival, D.E., Wood, D.W.: Forces and moments on flat plates of small aspect ratio with application to $\mathrm{PV}$ wind loads and small wind turbine blades. Energies 8(4), 1-16 (2015)

13. Piatek, M.: Identification of the servo motor used in the walking robot. AUTOMATYKA 14(1) (2010)

14. Pusch, M., Ossmann, D., Luspay, T.: Structured control design for a highly flexible flutter demonstrator. Aerospace 6(3). https://doi.org/10.3390/aerospace6030027. https://www.mdpi. com/2226-4310/6/3/27 (2019)

15. Sendner, F.M., Stahl, P., Roessler, C., Hornung, M.: Designing an uav propulsion system for dedicated acceleration and deceleration requirements. AIAA AVIATION Forum. American Institute of Aeronautics and Astronautics. https://doi.org/10.2514/6.20174105 (2017)

16. Sendner, F.M., Stahl, P., Roessler, C., Hornung, M.: Design and testing of an electric actuated airbrake for dynamic airspeed control of an unmanned aeroelastic research vehicle. AIAA AVIATION Forum. American Institute of Aeronautics and Astronautics. https://doi.org/10.2514/6.2018-3194 (2018)

17. Takarics, B., Patartics, B., Luspay, T., Vanek, B., Roessler, C., Bartasevicius, J., Koeberle, S.J., Hornung, M., Teubl, D., Pusch, M., Wustenhagen, M., Kier, T.M., Looye, G., Bauer, P., Meddaikar, Y.M., Waitman, S., Marcos, A.: Active flutter mitigation testing on the FLEXOP demonstrator aircraft. In: AIAA Scitech
2020 Forum (2020). https://doi.org/10.2514/6.2020-1970. https:// arc.aiaa.org/doi/abs/10.2514/6.2020-1970

18. Wada, T., Ishikawa, M., Kitayoshi, R., Maruta, I., Sugie, T.: Practical modeling and system identification of R/C servo motors. In: 2009 IEEE Control Applications, (CCA) Intelligent Control, (ISIC), pp. 1378-1383 (2009). https://doi.org/10.1109/CCA.2009. 5280987

Publisher's Note Springer Nature remains neutral with regard to jurisdictional claims in published maps and institutional affiliations.

Peter Bauer received his M.Sc. and Ph.D in Mechanical Engineering (Aerospace Engineering) in 2005 and 2013, respectively, at Budapest University of Technology and Economics, Budapest, Hungary. He is a Senior Research Fellow at the Institute for Computer Science and Control, Budapest, Hungary. He is quality manager in EU H2020 projects (Flexop, FLiPASED) and was actively involved in the VISION EU H2020 project regarding camera-based landing (IMU-camera data fusion) and camera, GPS, ILS system fault detection research. His other research fields cover state and parameter estimation, tracking control design, aircraft autopilot and sense and avoid system development. He also designs and coordinates flight test experiences.

Lysandros Anastasopoulos earned his B.Sc. and M.Sc. in Mechanical Engineering at the Technical University of Darmstadt. After a short time in the industry, he worked as a research assistant at the Technical University of Munich in the field of real-time aircraft systems testing. $\mathrm{He}$ is currently employed in Switzerland's aviation industry and collaborates with the National Technical University of Athens in the field of non-linear dynamics.

Franz-Michael Sendner received his M.Sc. and B.Sc. degree in Aerospace Engineering from Technical University of Munich (TUM). He joined the Institute of Aircraft Design of TUM as a research scientist in 2016, being in charge of the propulsion and airbrake system of the FLEXOP demonstrator. In 2018 Franz Sendner joined FACC's Research \& Technology Department, starting with the demonstrator wing manufacturing and currently working on integral design based solutions for wing moveables of transport aircraft.

Mirko Hornung received his doctorate in aeronautical engineering from the University of the Bundeswehr (Federal Armed Forces) in Munich. His thesis on reusable space transport systems was awarded a research prize in 2003. From 2003 to 2009 he worked at EADS Defence and Security (today: Airbus Defence and Space) on propulsion system integration, program management and business development. In his last position, he was responsible for the initial development of future airvehicles and unmanned aerial systems. He was appointed Professor for Aircraft Design at TUM in 2010 and is head of the Institute of Aircraft Design and his research is in the area of aircraft design, integration, and evaluation. His focus is on conceptual aircraft design and the theoretical and experimental evaluation of overall system technologies under operational conditions. $\mathrm{He}$ is Executive Director Research and Technology of Bauhaus Luftfahrt e.V., a non-profit organization and an internationally oriented think tank. Mirko Hornung is senior member of AIAA, member of the executive board of Deutsche Gesellschaft für Luft- und Raumfahrt (DGLR), member of the bavAIRia advisory board and member of the Transport Advisory Group for H2020.

Balint Vanek currently deputy head of the research laboratory SCL at SZTAKI, obtained his Msc from Budapest University of Technology and Economics in 2003 and the $\mathrm{PhD}$ from University of Minnesota in 
2008. Prior to working at SZTAKI he was a senior research scientist at Honeywell Aerospace Advanced Technology. Bálint's research interest includes guidance, navigation and control related to safety aspects of both manned and unmanned aerial vehicles, including fault detection and reconfigurable control of aerospace systems, unmanned aerial vehicles (UAV) and their insertion into the common airspace, as well as aeroservoelastic behavior of advanced aircraft configurations. He is the coordinator of the FLEXOP and FLiPASED EU H2020 projects. 